\title{
Thermal right-handed neutrino production rate in the non-relativistic regime
}

\section{Laine and Y. Schröder}

Faculty of Physics, University of Bielefeld, D-33501 Bielefeld, Germany

E-mail: laine@physik.uni-bielefeld.de, yorks@physik.uni-bielefeld.de

ABSTRACT: We consider the next-to-leading order thermal production rate of heavy righthanded neutrinos in the non-relativistic regime $m_{\text {top }} \lesssim \pi T \ll M$, where $m_{\text {top }}$ refers to the electroweak scale. Rephrasing the problem in an OPE language and making use of different techniques than a previous analysis by Salvio et al, we confirm the general structure of their result and many of the coefficients. We also extend the analysis to the next order in the nonrelativistic expansion, thereby revealing the leading non-trivial momentum dependence, as well as to NNLO in couplings, revealing the leading sensitivity to thermal resummations. Our results are expressed as a sum of simple "master" structures, which renders them a suitable starting point for determining the next-to-leading order rate also in the relativistic regime $\pi T \sim M$.

KEywords: Thermal Field Theory, NLO Computations, Neutrino Physics

ARXIV EPRINT: 1112.1205 


\section{Contents}

1 Introduction 1

2 Setup 2

3 Issues with regularization 4

4 Leading order at zero temperature 5

5 General method at non-zero temperature $\quad 6$

5.1 Wick contractions 6

$\begin{array}{ll}5.2 & \text { Single-pole cut }\end{array}$

5.3 Double-pole cut 8

5.4 Putting everything together 9

6 Next-to-leading order analysis $\quad 10$

$\begin{array}{lll}7 & \text { Next-to-leading order results } & 11\end{array}$

8 OPE representation $\quad 12$

9 Conclusions $\quad 14$

$\begin{array}{lr}\text { A Definitions of master sum-integrals } & 15\end{array}$

$\begin{array}{ll}\text { B Results for master spectral functions } & 17\end{array}$

$\begin{array}{lr}\text { C Euclidean large-momentum expansions } & 18\end{array}$

D On the treatment of Dirac traces $\quad 20$

\section{Introduction}

Weakly interacting particles produced by scatterings taking place in a hot plasma could conceivably play a role in cosmology. For instance, some Dark Matter candidates, such as axions, axinos, gravitinos, or sterile neutrinos, could originate this way (for reviews see e.g. refs. $[1,2])$. Another example is the so-called Leptogenesis model for explaining the Baryon Asymmetry of the Universe [3], in which thermally produced out-of-equilibrium right-handed neutrinos could act as one of the main building blocks.

Although the Leptogenesis scenario has been thoroughly studied (for reviews, see e.g. refs. $[4,5])$, there are some ingredients in the analysis that do not appear to be on a sound theoretical footing. In particular, it has recently been pointed out that in the 
"ultrarelativistic" regime, meaning temperatures much higher than the mass of the righthanded neutrinos, $\pi T \gg M \gtrsim m_{\text {top }}$, the expressions that had been used are not correct even at leading order in the coupling constants (because of infrared sensitivity infinitely many loop orders contribute to the leading-order result in this kinematic regime), and that they had therefore underestimated the production rate [6]. On the other hand, in another recent contribution, it was shown that in the "non-relativistic regime", $m_{\text {top }} \ll \pi T \ll M$, the significance of loop effects had probably been overestimated in the literature, because they had been only partly accounted for, whereby an important cancellation was missed [7]. (More complicated issues like CP-violation could contain even larger uncertainties [8]-[13].)

In view of the mentioned developments, it might be useful to revisit the analysis of the right-handed neutrino production rate also in the "relativistic" regime, $m_{\text {top }} \lesssim \pi T \sim M$, checking the importance of loop corrections through an explicit computation. Here, we take a first step in this direction, by formulating the setup of a next-to-leading order (NLO) analysis, and by carrying it out in the non-relativistic regime previously considered in ref. [7]. Given that the analysis of ref. [7] is exceedingly complicated, our goal was to reproduce its results by simpler methods, and also to extend them up to a higher order in an expansion in $(\pi T / M)^{2}$.

It is important to understand that when discussing a non-relativistic regime, we place ourselves in the position of the particles produced, with an invariant mass $\mathcal{K}^{2}=$ $M^{2} \gg(\pi T)^{2}$, a kinetic energy $k^{2} /(2 M) \sim \pi T$, and therefore an average velocity $k / M \sim$ $\sqrt{\pi T / M} \ll 1$. From the plasma perspective, however, the four-momentum $\mathcal{K}$ is an external probe, which has no dynamical effect. It simply represents a "hard" external scale, with $k^{0}, k,\left|k^{0} \pm k\right| \gg \pi T$. A proper tool for addressing this kinematic situation is the Operator Product Expansion (OPE) [14], formulated within the context of thermal field theory only rather recently [15], and subsequently applied e.g. to correlators of the energy-momentum tensor of a Yang-Mills plasma [16, 17]. Understanding the computation in this language has significant conceptual benefits, for instance by leading to a general framework for analyzing the infrared (IR) sensitivity of the results [15], a topic that remains otherwise to be studied empirically [7].

\section{Setup}

We consider a plasma made of Standard Model particles, in thermal equilibrium at a temperature $T$, and interacting with right-handed neutrinos, of mass $M$, through Yukawa interactions, parametrized by a coupling $h_{\nu}$. (It would be natural to assume the Yukawa couplings to build a $3 \times 3$ matrix, but our results are flavour-blind so we can simplify the notation without a loss of generality.) As long as the density of the right-handed neutrinos is below the equilibrium value, their production rate can be computed from first principles using a linear response or Kubo type analysis. Then, essentially, the production rate is determined by the cut (or imaginary part) of the self-energy of the right-handed neutrinos.

An explicit derivation of the production rate has been presented in ref. [18]. ${ }^{1}$ Letting

\footnotetext{
${ }^{1}$ The notation in ref. [18] assumed a broken electroweak symmetry, but the derivation goes through also with a dynamical doublet. The derivation was extended to $\mu_{\ell} \neq 0$ in ref. [19].
} 
$\tilde{\phi} \equiv i \sigma_{2} \phi^{*}$; denoting by $\ell$ a lepton doublet; and by $a_{\mathrm{L}}, a_{\mathrm{R}}$ the left and right projectors, $a_{\mathrm{L}} \equiv\left(1-\gamma_{5}\right) / 2, a_{\mathrm{R}} \equiv\left(1+\gamma_{5}\right) / 2$, the differential production rate reads

$$
\frac{\mathrm{d} N(\mathcal{K})}{\mathrm{d}^{4} \mathcal{X} \mathrm{d}^{3} \mathbf{k}}=\frac{\left|h_{\nu \mathrm{B}}\right|^{2}}{(2 \pi)^{3} k^{0}} \operatorname{Tr}\left\{\not \mathcal{L} a_{\mathrm{L}}\left[n_{\mathrm{F}}\left(k^{0}-\mu_{\ell}\right) \rho(\mathcal{K})+n_{\mathrm{F}}\left(k^{0}+\mu_{\ell}\right) \rho(-\mathcal{K})\right] a_{\mathrm{R}}\right\}+\mathcal{O}\left(\left|h_{\nu \mathrm{B}}\right|^{4}\right),
$$

where $h_{\nu \mathrm{B}}$ is the bare neutrino Yukawa coupling; $n_{\mathrm{F}}\left(k^{0}\right) \equiv 1 /\left[\exp \left(k^{0} / T\right)+1\right]$ is the Fermi distribution; $\mu_{\ell}$ denotes a leptonic chemical potential; $\rho$ is the spectral function related to the composite operator $\tilde{\phi}^{\dagger} \ell ; \mathcal{X}=\left(x^{0}, \mathbf{x}\right)$; and $\mathcal{K}=\left(k^{0}, \mathbf{k}\right)$ is an on-shell four-momentum, with $k^{0}=\sqrt{k^{2}+M^{2}}$. If the plasma is charge-symmetric, i.e. $\mu_{\ell}=0$, then $\rho$ is symmetric in $\mathcal{K} \rightarrow-\mathcal{K}$ and the two terms can be combined; we make this assumption in the following.

For a practical computation, it is convenient to employ Euclidean conventions. We define

$$
\Pi_{E}(K) \equiv\left|h_{\nu \mathrm{B}}\right|^{2} \operatorname{Tr}\left\{i K\left[\int_{0}^{\beta} \mathrm{d} \tau \int_{\mathbf{x}} e^{i K \cdot X}\left\langle\left(\tilde{\phi}^{\dagger} a_{\mathrm{L}} \ell\right)(X)\left(\bar{\ell} a_{\mathrm{R}} \tilde{\phi}\right)(0)\right\rangle_{T}\right]\right\}
$$

where now $X=(\tau, \mathbf{x})$; the vector $K=\left(k_{n}, \mathbf{k}\right)$ is Euclidean, with $k_{n}=(2 n+1) \pi T, n \in \mathbb{Z}$; and $\langle\ldots\rangle_{T}$ refers to a thermal expectation value. Defining a differential "decay rate", $\Gamma$, in accordance with ref. [7] as

$$
\frac{\mathrm{d} N(\mathcal{K})}{\mathrm{d}^{4} \mathcal{X} \mathrm{d}^{3} \mathbf{k}} \equiv \frac{2 n_{\mathrm{F}}\left(k^{0}\right)}{(2 \pi)^{3}} \Gamma(\mathcal{K})
$$

then eqs. (2.1), (2.2) together with the standard relation between the Euclidean correlator and the spectral function $\left(\rho=\operatorname{Im} \Pi_{R}=\operatorname{Im}\left\{\Pi_{E}\right\}_{k_{n} \rightarrow-i\left[k^{0}+i 0^{+}\right]}\right.$, cf. e.g. ref. [20]) imply that

$$
\Gamma(\mathcal{K})=\frac{1}{k^{0}} \operatorname{Im}\left\{\Pi_{E}(K)\right\}_{k_{n} \rightarrow-i\left[k^{0}+i 0^{+}\right]} .
$$

Here Im refers to a discontinuity, or cut, across the real $k^{0}$-axis, and the relation is valid even on the non-perturbative level.

To compute the correlator of eq. (2.2), let us establish conventions for the relevant part of the Standard Model. The Higgs field interacts according to the Euclidean Lagrangian

$$
\mathcal{L}_{\phi}=\left(D_{\mu} \phi\right)^{\dagger}\left(D_{\mu} \phi\right)+m_{\mathrm{B}}^{2} \phi^{\dagger} \phi+\lambda_{\mathrm{B}}\left(\phi^{\dagger} \phi\right)^{2}+h_{t \mathrm{~B}} \bar{q} a_{\mathrm{R}} \tilde{\phi} t+h_{t \mathrm{~B}}^{*} \bar{t} \tilde{\phi}^{\dagger} a_{\mathrm{L}} q
$$

The mass parameter, $m_{\mathrm{B}}^{2}$, will mostly be omitted because we assume that $\pi T \gtrsim m_{\text {top }}$ and, as already alluded to above, the NLO results turn out to be IR safe in this regime (cf. section 8). Technically the calculation is performed as if we were in the symmetry restored phase although, as will become clear later on, this assumption can be modestly relaxed. When acting on the Higgs the covariant derivative takes the form

$$
\left[D_{\mu} \phi\right]_{m}=\left(\delta_{m n} \partial_{\mu}-i g_{2 \mathrm{~B}} T_{m n}^{a} A_{\mu}^{a}+i g_{1 \mathrm{~B}} T_{m n}^{0} B_{\mu}\right) \phi_{n},
$$

where $m, n \in\{1,2\} ; A_{\mu}^{a}, B_{\mu}$ are the $\mathrm{SU}_{\mathrm{L}}(2)$ and $\mathrm{U}_{\mathrm{Y}}(1)$ gauge fields, respectively; $T^{a}$ are Hermitean generators of $\mathrm{SU}_{\mathrm{L}}(2)$, normalized as $\operatorname{Tr}\left[T^{a} T^{b}\right]=\frac{1}{2} \delta^{a b} ; T_{m n}^{0} \equiv \frac{1}{2} \delta_{m n}$; and the 
couplings appearing are bare ones. The quark Yukawa interaction in eq. (2.5) contains the doublet $q=(t b)^{T}$. The leptons interact according to the Euclidean Lagrangian

$$
\mathcal{L}_{\ell}=\bar{\ell} \gamma_{\mu} D_{\mu} \ell
$$

where $\ell=(\nu e)^{T}$. When acting on the lepton doublet, the covariant derivative reads

$$
\left[D_{\mu} \ell\right]_{m}=\left(\delta_{m n} \partial_{\mu}-i\left[g_{2 \mathrm{~B}} T_{m n}^{a} A_{\mu}^{a}+g_{1 \mathrm{~B}} T_{m n}^{0} B_{\mu}\right] a_{\mathrm{L}}-i\left[g_{1 \mathrm{~B}} \delta_{m 2} \delta_{n 2} B_{\mu}\right] a_{\mathrm{R}}\right) \ell_{n} .
$$

\section{Issues with regularization}

A loop computation in quantum field theory necessitates regularization, and by far the most convenient choice for this is the dimensional one, which we also adopt here. Unfortunately, with chiral gauge theories dimensional regularization leads to inevitable problems. Although well-known, we briefly remark on some of the issues in this section.

In $D$ dimensions, the normal (Euclidean) Dirac matrices can be taken to satisfy

$$
\left\{\gamma_{\mu}, \gamma_{\nu}\right\}=2 g_{\mu \nu} \equiv 2 \delta_{\mu \nu}, \quad \gamma_{\mu}^{\dagger}=\gamma_{\mu}, \quad \operatorname{Tr}(1)=4
$$

According to the 't Hooft - Veltman $[21,22]$ convention a Hermitean $\gamma_{5}$ can be defined as

$$
\gamma_{5} \equiv \gamma_{0} \gamma_{1} \gamma_{2} \gamma_{3}, \quad \gamma_{5}^{2}=1
$$

An important implication from here is that $[23,24]$

$$
a_{\mathrm{R}} \gamma_{\mu} a_{\mathrm{L}}=\tilde{\gamma}_{\mu} a_{\mathrm{L}}
$$

where

$$
\tilde{\gamma}_{\mu} \equiv\left\{\begin{array}{cc}
\gamma_{\mu}, & \mu \leq 3 \\
0, & \mu>3
\end{array} .\right.
$$

In the (free) kinetic part of eq. (2.7) we might then imagine defining a Lagrangian as $\bar{\ell} \gamma_{\mu} \partial_{\mu} \ell \stackrel{?}{\rightarrow} \bar{\ell} \tilde{\gamma}_{\mu} \partial_{\mu} \ell=\bar{\ell} a_{\mathrm{R}} \tilde{\gamma}_{\mu} \partial_{\mu} a_{\mathrm{L}} \ell+\bar{\ell} a_{\mathrm{L}} \tilde{\gamma}_{\mu} \partial_{\mu} a_{\mathrm{R}} \ell$, respecting gauge symmetry; but then the propagator is four-dimensional and not properly regularized. Or, we could keep the regularized form,

$$
\begin{aligned}
\bar{\ell} \gamma_{\mu} \partial_{\mu} \ell & =\bar{\ell}\left(a_{\mathrm{R}}+a_{\mathrm{L}}\right) \gamma_{\mu} \partial_{\mu}\left(a_{\mathrm{R}}+a_{\mathrm{L}}\right) \ell \\
& =\bar{\ell} a_{\mathrm{R}} \gamma_{\mu} \partial_{\mu} a_{\mathrm{L}} \ell+\bar{\ell} a_{\mathrm{R}} \gamma_{\mu} \partial_{\mu} a_{\mathrm{R}} \ell+\bar{\ell} a_{\mathrm{L}} \gamma_{\mu} \partial_{\mu} a_{\mathrm{L}} \ell+\bar{\ell} a_{\mathrm{L}} \gamma_{\mu} \partial_{\mu} a_{\mathrm{R}} \ell,
\end{aligned}
$$

leading to the usual $D$-dimensional propagator; but then gauge symmetry is (slightly) broken by the coupling of the different chiralities. Though extremely naive, these remarks already illustrate some of the complications encountered. Still, consistent computations are possible if counterterms and operator mixing are properly accounted for [23, 24], and a closely related practical recipe has also been put forward [25].

In the prescription of ref. [25], one can conveniently work with $D$-dimensional Dirac matrices after defining axial-vector currents with the structure $\gamma_{\mu} \gamma_{5} \rightarrow \frac{1}{2}\left[\gamma_{\mu}, \gamma_{5}\right]=$ 
$\frac{1}{3 !} \varepsilon_{\mu \nu \rho \sigma} \gamma_{\nu} \gamma_{\rho} \gamma_{\sigma}$, pulling the antisymmetric tensors $\varepsilon$ outside the actual integrals and utilizing only their antisymmetry inside the traces. The latter is made explicit by writing

$$
\frac{1}{2}\left[\gamma_{\mu}, \gamma_{5}\right]=\frac{1}{12} \varepsilon_{\mu \nu \rho \sigma}\left(\gamma_{\nu} \gamma_{\rho} \gamma_{\sigma}-\gamma_{\sigma} \gamma_{\rho} \gamma_{\nu}\right)
$$

As a further simplification, it has been argued in ref. [25] that a naively anticommuting $\gamma_{5}$ in combination with $\gamma_{5}^{2}=1$ can be used in fermionic traces containing more than one $\gamma_{5}$, with the exception of closed fermion loops. The consistency of this prescription has been verified up to 3-loop order in connection with singlet as well as non-singlet axialvector operators in QCD [25, 26]. We will employ this recipe as a "minimal crosscheck", commenting also on how it differs from the 't Hooft - Veltman scheme when closed fermion loops are present.

It is important to realize, however, that in the computation of the spectral function according to eq. (2.4) some of the ambiguities affecting the Euclidean correlator drop out. Indeed, a non-zero cut arises from logarithms or, in the context of dimensional regularization, from terms containing $1 / \epsilon$-poles (cf. eq. (B.3)). So, in terms of the Euclidean correlator, it is only necessary to get the $1 / \epsilon$-poles correct. Of course, the bare parameters also bring along $1 / \epsilon$-poles, so lower-order graphs need to be worked out to a higher depth in the $\epsilon$-expansion.

\section{Leading order at zero temperature}

In order to get going, we start by an almost trivial step, performing a leading order analysis at zero temperature. This has the lucky feature of showing that at leading order, we are free from the subtleties of section 3 to all orders in $\epsilon$.

The starting point is to carry out Wick contractions in eq. (2.2). Then, although eq. (3.3) implies that the external momentum appears as

$$
a_{\mathrm{R}} \not K a_{\mathrm{L}}=\tilde{K} a_{\mathrm{L}}
$$

rotational symmetry guarantees that we are free to choose $K$ to have at most $\min (D-1,3)$ non-zero spatial components. With this choice, $\tilde{K} a_{\mathrm{L}}=\not K a_{\mathrm{L}}$, and

$$
\operatorname{Tr}\left[i \not K a_{\mathrm{L}}(i \not K-i \not P) a_{\mathrm{R}}\right]=2 \tilde{K} \cdot(\tilde{P}-\tilde{K})=2 K \cdot(P-K) .
$$

So we have the same expression as in Naive Dimensional Regularization (NDR), in which it is assumed, despite algebraic inconsistencies, that $\gamma_{5}$ anticommutes with all $\gamma_{\mu}$.

With eq. (4.2) at hand, a few steps lead to

$$
\Pi_{E}(K)=4\left|h_{\nu \mathrm{B}}\right|^{2} \oiint_{P} \frac{K \cdot(P-K)}{P^{2}(P-K)^{2}}=2\left|h_{\nu \mathrm{B}}\right|^{2} \oint_{P}\left[\frac{1}{(P-K)^{2}}-\frac{1}{P^{2}}-\frac{K^{2}}{P^{2}(P-K)^{2}}\right],
$$

where the additional factor 2 comes from the isospin trace, and we completed squares in the numerator. At zero temperature, with $\mathscr{\&}_{P} \rightarrow \int_{P}$, only the last term contributes, and 
we obtain ${ }^{2}$

$$
\Pi_{E}^{(0)}(K)=-2\left|h_{\nu \mathrm{B}}\right|^{2} K^{2} \frac{\mu^{-2 \epsilon}}{(4 \pi)^{2}}\left(\frac{1}{\epsilon}+\ln \frac{\bar{\mu}^{2}}{K^{2}}+2+\mathcal{O}(\epsilon)\right) .
$$

Subsequently, eq. (2.4) yields

$$
\Gamma^{(0)}(\mathcal{K})=\frac{\left|h_{\nu \mathrm{B}}\right|^{2} \mathcal{K}^{2}}{8 \pi k^{0}}+\mathcal{O}(\epsilon)
$$

where we have denoted $\mathcal{K}^{2} \equiv\left(k^{0}\right)^{2}-k^{2}$; and the Euclidean four-momentum transforms as $K^{2}=k_{n}^{2}+k^{2} \rightarrow-\mathcal{K}^{2}-i \operatorname{sign}\left(k^{0}\right) 0^{+}$. The result in eq. (4.5) agrees with eq. (3) of ref. [7].

For later reference, let us verify eq. (4.3) using the prescription introduced below eq. (3.6). Performing the isospin trace, but keeping the Dirac trace for the moment, we get

$$
\Pi_{E}(K)=-2\left|h_{\nu \mathrm{B}}\right|^{2} \operatorname{Tr}\left[\gamma_{\mu} a_{\mathrm{L}} \gamma_{\nu} a_{\mathrm{R}}\right] \sum_{P} \frac{K_{\mu}(K-P)_{\nu}}{P^{2}(P-K)^{2}} .
$$

Now, noting that $\operatorname{Tr}\left(\gamma_{\mu} a_{\mathrm{L}} \gamma_{\nu} a_{\mathrm{R}}\right)=\frac{1}{4} \operatorname{Tr}\left\{\gamma_{\mu}\left(\gamma_{\nu}-\gamma_{5} \gamma_{\nu} \gamma_{5}\right)+\gamma_{\mu}\left[\gamma_{\nu}, \gamma_{5}\right]\right\}$, the commutator part leads to $\frac{1}{24} \varepsilon_{\nu \rho \sigma \kappa} \operatorname{Tr}\left[\gamma_{\mu} \gamma_{\rho} \gamma_{\sigma} \gamma_{\kappa}-\gamma_{\mu} \gamma_{\kappa} \gamma_{\sigma} \gamma_{\rho}\right]$ and vanishes once the trace is expressed as a product of metric tensors. In the first part, the rule of ref. [25] with two $\gamma_{5}$ 's leads immediately to $\operatorname{Tr}\left[\gamma_{\mu} a_{\mathrm{L}} \gamma_{\nu} a_{\mathrm{R}}\right] \rightarrow 2 g_{\mu \nu}$, agreeing with eq. (4.3). In contrast, a strict use of eq. (3.2) with two $\gamma_{5}$ 's leads to the middle equation in eq. (4.2) and necessitates a further argument.

\section{General method at non-zero temperature}

Proceeding to NLO, we illustrate some details by computing explicitly the "Higgs correction", i.e. terms proportional to $\lambda$.

\subsection{Wick contractions}

Carrying out the Wick contractions and making use of eq. (4.2) we obtain, in analogy with eq. (4.3),

$$
\begin{aligned}
\Pi_{E}^{(\lambda)} & =24\left|h_{\nu \mathrm{B}}\right|^{2} \lambda_{\mathrm{B}} \oiint_{P, Q} \frac{K \cdot(K-P)}{Q^{2} P^{4}(K-P)^{2}} \\
& =12\left|h_{\nu \mathrm{B}}\right|^{2} \lambda_{\mathrm{B}} \oint_{P, Q} \frac{K^{2}+(K-P)^{2}-P^{2}}{Q^{2} P^{4}(K-P)^{2}} \\
& =12\left|h_{\nu \mathrm{B}}\right|^{2} \lambda_{\mathrm{B}} \oint_{P, Q} \frac{1}{Q^{2}}\left[\frac{1}{P^{4}}-\frac{1}{P^{2}(K-P)^{2}}+\frac{K^{2}}{P^{4}(K-P)^{2}}\right] .
\end{aligned}
$$

The factorized structures have well-known expressions in dimensional regularization [27]:

$$
\begin{aligned}
& \mathcal{F}_{Q} \frac{1}{Q^{2}}=\int_{\mathbf{q}} \frac{n_{\mathrm{B}}(q)}{q}=\frac{T^{2} \mu^{-2 \epsilon}}{12}\left\{1+2 \epsilon\left[\ln \left(\frac{\bar{\mu}}{4 \pi T}\right)+1+\frac{\zeta^{\prime}(-1)}{\zeta(-1)}\right]+\mathcal{O}\left(\epsilon^{2}\right)\right\}, \\
& \mathcal{F}_{P} \frac{1}{P^{4}}=\frac{1-2 \epsilon}{2} \int_{\mathbf{p}} \frac{n_{\mathrm{B}}(p)}{p^{3}}=\frac{\mu^{-2 \epsilon}}{(4 \pi)^{2}}\left[\frac{1}{\epsilon}+2 \ln \left(\frac{\bar{\mu} e^{\gamma_{\mathrm{E}}}}{4 \pi T}\right)+\mathcal{O}(\epsilon)\right]
\end{aligned}
$$

\footnotetext{
${ }^{2}$ The $\overline{\mathrm{MS}}$ scale parameter is introduced in a usual way, inserting $1=\mu^{-2 \epsilon} \bar{\mu}^{2 \epsilon} \frac{\exp \left(\gamma_{\mathrm{E}} \epsilon\right)}{(4 \pi)^{\epsilon}}$.
} 
where $n_{\mathrm{B}}(p) \equiv 1 /[\exp (p / T)-1]$ denotes the Bose distribution, and a partial integration was carried out for obtaining the first representation of the latter term. (It is worth noting that this term has a power-like IR divergence which is not visible in dimensional regularization; the $1 / \epsilon$ has an ultraviolet (UV) origin. Nevertheless, as long as dimensional regularization is applied consistently, there is no reason to worry; we return to this in the paragraph following eq. (5.15), and a comprehensive analysis is presented in section 8.) However, the product of eqs. (5.2), (5.3) is $K$-independent, so there is no cut, and no contribution to $\Gamma(\mathcal{K})$.

\section{$5.2 \quad$ Single-pole cut}

Non-trivial cuts arise from the 2nd and 3rd terms of eq. (5.1). Starting with the second term, the Matsubara sum can be carried out exactly, yielding

$$
\sum_{P} \frac{1}{P^{2}(K-P)^{2}}=\int_{P} \frac{1}{P^{2}(K-P)^{2}}+\int_{\mathbf{p}} \frac{n_{\mathrm{B}}(p)-n_{\mathrm{F}}(p)}{p}\left[\frac{1}{(K-P)^{2}}\right]_{P},
$$

where the notation $[\ldots]_{P} \equiv \frac{1}{2} \sum_{p_{n}= \pm i p}(\ldots)$ corresponds to setting the thermal line onshell; and the integration variable was renamed in the fermionic cut. The vacuum term has the familiar structure of eq. (4.4), with a cut $\frac{1}{16 \pi}$, whereas the thermal part can formally be expanded in $p$, given that the $p$-integration is exponentially convergent due to the thermal distributions:

$$
\left[\frac{1}{(K-P)^{2}}\right]_{P}=\left[\frac{1}{K^{2}-2 K \cdot P}\right]_{P}=\left[\frac{1}{K^{2}}+\frac{2 K \cdot P}{K^{4}}+\frac{4(K \cdot P)^{2}}{K^{6}}+\ldots\right]_{P} .
$$

This yields an OPE-type expansion. However, as long as we stay away from the light-cone $\left(\operatorname{Re}\left[K^{2}\right]_{k_{n} \rightarrow-i\left[k^{0}+i 0^{+}\right]} \neq 0\right)$, this expansion has no cut. Therefore, a contribution only arises from the vacuum part of eq. (5.4), multiplying eq. (5.2):

$$
\operatorname{Im}\left\{\sum_{P, Q} \frac{1}{Q^{2} P^{2}(K-P)^{2}}\right\}_{k_{n} \rightarrow-i\left[k^{0}+i 0^{+}\right]}=\frac{1}{16 \pi} \frac{T^{2}}{12}+\mathcal{O}(\epsilon) .
$$

It is useful for later reference to repeat the analysis of the thermal part in a more detailed way. For this purpose we set $\mathbf{k}=\mathbf{0}$ and work out the cut exactly. Given that for $k=0$

$$
\left[\frac{1}{(K-P)^{2}}\right]_{P ; k=0}=\frac{1}{2 k_{n}}\left(\frac{1}{k_{n}-2 i p}+\frac{1}{k_{n}+2 i p}\right)=\frac{1}{4 i p}\left(\frac{1}{k_{n}-2 i p}-\frac{1}{k_{n}+2 i p}\right),
$$

we get

$$
\operatorname{Im}\left\{\left[\frac{1}{(K-P)^{2}}\right]_{P ; k=0}\right\}_{k_{n} \rightarrow-i\left[k^{0}+i 0^{+}\right]}=\frac{\pi}{4 p}\left[\delta\left(k^{0}-2 p\right)-\delta\left(k^{0}+2 p\right)\right] .
$$

So, for $k^{0}=\sqrt{k^{2}+M^{2}}>0$,

$$
\begin{aligned}
\operatorname{Im}\left\{\int_{\mathbf{p}} \frac{n_{\mathrm{B}}(p)-n_{\mathrm{F}}(p)}{p}\left[\frac{1}{(K-P)^{2}}\right]_{P ; k=0}\right\} & =\frac{1}{8 \pi} \int_{0}^{\infty} \mathrm{d} p\left[n_{\mathrm{B}}(p)-n_{\mathrm{F}}(p)\right] \delta\left(k^{0}-2 p\right) \\
& =\frac{1}{16 \pi}\left[n_{\mathrm{B}}\left(\frac{k^{0}}{2}\right)-n_{\mathrm{F}}\left(\frac{k^{0}}{2}\right)\right] .
\end{aligned}
$$

In other words we do find a correction to the $\frac{1}{16 \pi}$ appearing in eq. (5.6) but it is exponentially small, suppressed by $e^{-k^{0} / T}$ in the regime $k^{0} \gg T$ that we are interested in. 


\subsection{Double-pole cut}

It remains to work out the 3rd term of eq. (5.1). This is slightly less trivial than the 2 nd term because of the appearance of the double pole. Nevertheless the basic point is the same: denoting $E_{p} \equiv p, E_{k p} \equiv|\mathbf{k}-\mathbf{p}|$, we get

$$
\begin{aligned}
\mathcal{F}_{P} & \frac{1}{P^{4}(K-P)^{2}} \\
= & \int_{\mathbf{p}}\left(-\frac{\partial}{\partial E_{p}^{2}}\right) T \sum_{p_{n}} \frac{1}{\left(p_{n}^{2}+E_{p}^{2}\right)\left[\left(k_{n}-p_{n}\right)^{2}+E_{k p}^{2}\right]} \\
= & \int_{\mathbf{p}}\left(-\frac{\partial}{\partial E_{p}^{2}}\right)\left\{\int_{-\infty}^{\infty} \frac{\mathrm{d} p_{0}}{2 \pi} \frac{1}{\left(p_{0}^{2}+E_{p}^{2}\right)\left[\left(k_{n}-p_{0}\right)^{2}+E_{k p}^{2}\right]}\right. \\
& \left.\quad+\frac{n_{\mathrm{B}}\left(E_{p}\right)}{E_{p}}\left[\frac{1}{\left(k_{n}-p_{n}\right)^{2}+E_{k p}^{2}}\right]_{P}-\frac{n_{\mathrm{F}}\left(E_{k p}\right)}{E_{k p}}\left[\frac{1}{p_{n}^{2}+E_{p}^{2}}\right]_{K-P}\right\} \\
= & \int_{P} \frac{1}{P^{4}(K-P)^{2}}+\int_{\mathbf{p}}\left\{-\frac{\partial}{\partial E_{p}^{2}} \frac{n_{\mathrm{B}}\left(E_{p}\right)}{E_{p}}+\frac{n_{\mathrm{F}}\left(E_{p}\right)}{E_{p}} \frac{\partial}{\partial E_{k p}^{2}}\right\}\left[\frac{1}{\left(k_{n}-p_{n}\right)^{2}+E_{k p}^{2}}\right]_{P},
\end{aligned}
$$

where we substituted $\mathbf{p} \rightarrow \mathbf{k}-\mathbf{p}$ in the last term. The thermal part is more complicated than before, but it is clear that it can again be expanded in inverse powers of $1 / K^{2}$, and all resulting integrals are exponentially convergent in the UV (in the IR there are divergences like in eq. (5.3) but this only leads to logarithms of $T$, not of $K$ ). Like in eq. (5.5), these terms give no cuts. The zero-temperature term, on the other hand, evaluates in dimensional regularization to

$$
\int_{P} \frac{1}{P^{4}(K-P)^{2}}=-\frac{K^{-2 \epsilon}}{\epsilon K^{2}} \frac{\Gamma(1+\epsilon) \Gamma^{2}(1-\epsilon)}{(4 \pi)^{2-\epsilon} \Gamma(1-2 \epsilon)}=-\frac{\mu^{-2 \epsilon}}{(4 \pi)^{2} K^{2}}\left(\frac{1}{\epsilon}+\ln \frac{\bar{\mu}^{2}}{K^{2}}\right)+\mathcal{O}(\epsilon) .
$$

Here $1 / \epsilon$ is an IR divergence, but the cut is IR finite, and can be extracted like in eq. (4.4). In total, then,

$$
\operatorname{Im}\left\{f_{P, Q} \frac{K^{2}}{Q^{2} P^{4}(K-P)^{2}}\right\}_{k_{n} \rightarrow-i\left[k^{0}+i 0^{+}\right]}=-\frac{1}{16 \pi} \frac{T^{2}}{12}+\mathcal{O}(\epsilon) .
$$

For the benefit of a reader nerved by the free use of dimensional regularization for handling IR divergences, let us repeat the derivation of eq. (5.12) in a more pedantic way, directly in $D=4$, avoiding IR divergences. Setting again $\mathbf{k}=\mathbf{0}$ for simplicity, and denoting $E_{0}^{2} \equiv p^{2}+m_{0}^{2}$, where $m_{0}^{2}>0$ is an IR regulator of the scalar line, we can rewrite eq. (5.10) as

$$
\begin{aligned}
& \sum_{P} \frac{1}{P^{4}(K-P)^{2}} \\
&=-\lim _{m_{0} \rightarrow 0} \frac{\mathrm{d}}{\mathrm{d} m_{0}^{2}} \int_{\mathbf{p}} T \sum_{p_{n}} \frac{1}{\left(p_{n}^{2}+E_{0}^{2}\right)\left[\left(k_{n}-p_{n}\right)^{2}+p^{2}\right]} \\
&=-\lim _{m_{0} \rightarrow 0} \frac{\mathrm{d}}{\mathrm{d} m_{0}^{2}} \int_{\mathbf{p}} \frac{1}{4 p E_{0}}\left\{\left[\frac{1}{i k_{n}+p+E_{0}}+\frac{1}{-i k_{n}+p+E_{0}}\right]\left[1-n_{\mathrm{F}}(p)+n_{\mathrm{B}}\left(E_{0}\right)\right]\right. \\
&\left.+\left[\frac{1}{i k_{n}+p-E_{0}}+\frac{1}{-i k_{n}+p-E_{0}}\right]\left[n_{\mathrm{F}}(p)+n_{\mathrm{B}}\left(E_{0}\right)\right]\right\} .
\end{aligned}
$$


Setting $k_{n} \rightarrow-i\left[k^{0}+i 0^{+}\right]$and taking the cut, the denominators turn into $\delta$-constraints like in eq. (5.8). If we choose $m_{0}<M$, only one of them can get realized:

$$
\begin{aligned}
\operatorname{Im}\left\{\sum_{P} \frac{k_{n}^{2}}{P^{4}(K-P)^{2}}\right\}_{k_{n} \rightarrow-i\left[k^{0}+i 0^{+}\right]} \\
\quad=\lim _{m_{0} \rightarrow 0} \frac{\mathrm{d}}{\mathrm{d} m_{0}^{2}} \int_{\mathbf{p}} \frac{\pi k_{0}^{2}}{4 p E_{0}} \delta\left(-k^{0}+p+E_{0}\right)\left[1-n_{\mathrm{F}}(p)+n_{\mathrm{B}}\left(E_{0}\right)\right] \\
\quad=\lim _{m_{0} \rightarrow 0} \frac{\mathrm{d}}{\mathrm{d} m_{0}^{2}}\left\{\frac{k_{0}^{2}-m_{0}^{2}}{16 \pi}\left[1-n_{\mathrm{F}}\left(\frac{k_{0}^{2}-m_{0}^{2}}{2 k_{0}}\right)+n_{\mathrm{B}}\left(\frac{k_{0}^{2}+m_{0}^{2}}{2 k_{0}}\right)\right]\right\} .
\end{aligned}
$$

Taking the derivative we do reproduce eq. (5.12), up to exponentially small terms.

\subsection{Putting everything together}

Inserting finally eqs. (5.6), (5.12) into eq. (5.1), we obtain

$$
\operatorname{Im}\left\{\Pi_{E}^{(\lambda)}\right\}_{k_{n} \rightarrow-i\left[k^{0}+i 0^{+}\right]}=\left|h_{\nu \mathrm{B}}\right|^{2} \lambda_{\mathrm{B}}\left(-\frac{T^{2}}{8 \pi}\right) .
$$

This corresponds to the Higgs contribution in eqs. (6.9), (7.2). ${ }^{3}$

We now return to the issue of IR divergences. As has been stressed in the introduction, from the point of view of the thermal medium the right-handed neutrino acts as a "hard probe"; any thermal effects on its production rate can be understood in the OPE language. But in the OPE language thermal effects amount to gauge-invariant condensates developing expectation values [15]. These, in turn, obtain IR divergences only at higher loop orders than considered here (this is checked explicitly in section 8). Therefore it is guaranteed, from general principles, that all IR divergences must cancel in the spectral function, and we are allowed to handle them through dimensional regularization, as long as it is applied consistently. Of course, the cancellation of IR divergences can also be checked directly [7]. At the current order, the cancellation in fact probably takes place in every "master" spectral function separately, and for an arbitrary value of $k^{0} / T$, as has been observed in the fully bosonic case [28].

Having thereby concluded the discussion of Higgs corrections, we note that similar methods work in all other cases as well. The full list of structures appearing can be found in appendix A, and the corresponding spectral functions are given in appendix B. Whereas for the current paper the cuts of appendix B are sufficient, in some contexts it may be desirable to also know the ultraviolet expansions in the full Euclidean domain (cf. e.g. section 8); these are listed in appendix $\mathrm{C}$, and have been derived with methods explained in refs. $[16,17]$.

\footnotetext{
${ }^{3}$ If the exponentially small terms are included, then eqs. (5.6), (5.9), (5.14) imply (for $\mathbf{k}=\mathbf{0}$ )

$$
\operatorname{Im}\left\{\Pi_{E}^{(\lambda)}\right\}=-\frac{\left|h_{\nu \mathrm{B}}\right|^{2} \lambda_{\mathrm{B}} T^{2}}{8 \pi}\left\{1+n_{\mathrm{B}}\left(\frac{k_{0}}{2}\right)-n_{\mathrm{F}}\left(\frac{k_{0}}{2}\right)-\frac{k_{0}}{4}\left[n_{\mathrm{B}}^{\prime}\left(\frac{k_{0}}{2}\right)+n_{\mathrm{F}}^{\prime}\left(\frac{k_{0}}{2}\right)\right]\right\} .
$$
}




\section{Next-to-leading order analysis}

Including now also contributions from the top quark and from gauge bosons, we turn to our full NLO expressions. In order to obtain a "universal" representation, we make use of completions of squares and substitutions of integration variables in order to express the results in terms of a minimal number of independent "master" sum-integrals, listed in appendix A. We specify graph-by-graph results in NDR in terms of these masters. ${ }^{4}$ Gauge parameter independence (with respect to both gauge groups) has been checked separately, so here only the Feynman gauge results are shown. They read

$$
\begin{aligned}
& \underbrace{\vdots \vdots \vdots}=12\left|h_{\nu \mathrm{B}}\right|^{2} \lambda_{\mathrm{B}}\left(-\mathcal{I}_{\mathrm{b}}+\mathcal{I}_{\mathrm{c}}+\mathcal{I}_{\mathrm{d}}\right) \text {, } \\
& =2\left|h_{\nu \mathrm{B}}\right|^{2}\left|h_{t \mathrm{~B}}\right|^{2} N_{\mathrm{c}}\left(2 \widetilde{\mathcal{I}}_{\mathrm{b}}-2 \widetilde{\mathcal{I}}_{\mathrm{c}}-2 \widetilde{\mathcal{I}}_{\mathrm{d}}+\widetilde{\mathcal{I}}_{\mathrm{e}}-\widetilde{\mathcal{I}}_{\mathrm{f}}+\widetilde{\mathcal{I}}_{\mathrm{h}}\right) \text {, }
\end{aligned}
$$

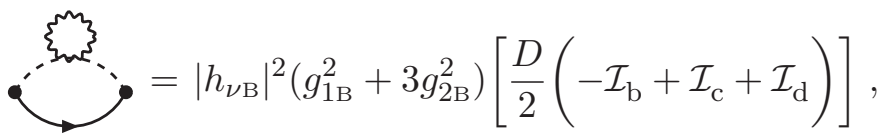

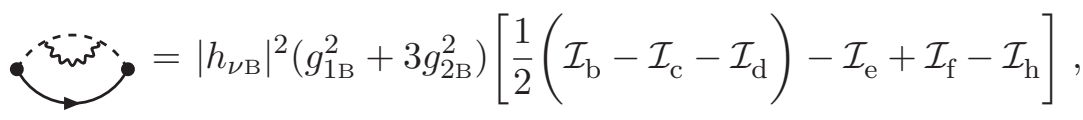

$$
\begin{aligned}
& \left.\underbrace{}_{\nu \mathrm{B}}\right|^{2}\left(g_{1 \mathrm{~B}}^{2}+3 g_{2 \mathrm{~B}}^{2}\right)\left[\frac{D-2}{2}\left(\mathcal{I}_{\mathrm{b}}-\widetilde{\mathcal{I}}_{\mathrm{b}}+\overline{\mathcal{I}}_{\mathrm{c}}-\widehat{\mathcal{I}}_{\mathrm{c}}+\widehat{\mathcal{I}}_{\mathrm{d}}-\overline{\mathcal{I}}_{\mathrm{d}}+\widehat{\mathcal{I}}_{\mathrm{h}},\right)\right] \text {, }
\end{aligned}
$$

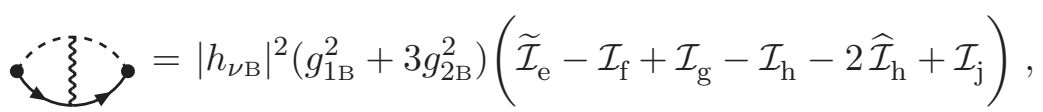

with dashed, solid, doubled, and wiggly lines representing scalars, leptons, quarks, and gauge bosons, respectively.

Inserting the cuts, or spectral functions, from appendix B; setting $D=4-2 \epsilon$; and renormalizing according to

$$
\begin{aligned}
\left|h_{\nu \mathrm{B}}\right|^{2} & =\left|h_{\nu}(\bar{\mu})\right|^{2} \mu^{2 \epsilon} \mathcal{Z}_{\nu}, \quad \text { with } \\
\mathcal{Z}_{\nu} & \equiv 1+\frac{1}{(4 \pi)^{2} \epsilon}\left[\left|h_{t}\right|^{2} N_{\mathrm{c}}-\frac{3}{4}\left(g_{1}^{2}+3 g_{2}^{2}\right)\right]+\mathcal{O}\left(g^{4}\right),
\end{aligned}
$$

where $g^{2}$ denotes a generic renormalized coupling, we obtain

$$
\begin{aligned}
\operatorname{Im} \Pi_{E}(\mathcal{K})=\frac{\left|h_{\nu}(\bar{\mu})\right|^{2} \mathcal{K}^{2}}{8 \pi}\left\{1-\frac{12 \lambda}{\mathcal{K}^{2}} \int_{\mathbf{p}} \frac{n_{\mathrm{B}}}{p}\right. \\
\quad-\left|h_{t}\right|^{2} N_{\mathrm{c}}\left[\frac{1}{(4 \pi)^{2}}\left(\ln \frac{\bar{\mu}^{2}}{\mathcal{K}^{2}}+\frac{7}{2}\right)+\frac{k_{0}^{2}+k^{2} / 3}{\mathcal{K}^{6}} \int_{\mathbf{p}} \frac{4 p n_{\mathrm{F}}}{3}\right]
\end{aligned}
$$

\footnotetext{
${ }^{4}$ We have checked that the same results are obtained, for every diagram, with the recipe described below eq. (3.6), whereas in the strict 't Hooft-Veltman scheme there are additional terms; cf. appendix D.
} 


$$
\begin{aligned}
& +\left(g_{1}^{2}+3 g_{2}^{2}\right)\left[\frac{3}{4(4 \pi)^{2}}\left(\ln \frac{\bar{\mu}^{2}}{\mathcal{K}^{2}}+\frac{29}{6}\right)+\frac{k_{0}^{2}+k^{2} / 3}{\mathcal{K}^{6}} \int_{\mathbf{p}} \frac{p\left(17 n_{\mathrm{F}}-16 n_{\mathrm{B}}\right)}{3}\right] \\
& \left.+\mathcal{O}\left(g^{4}, \frac{g^{3} T^{2}}{\mathcal{K}^{2}}, \frac{g^{2} T^{6}}{\mathcal{K}^{6}}\right)\right\} .
\end{aligned}
$$

We note in passing that the structure $k_{0}^{2}+k^{2} / 3$ originates from terms like the last one in eq. (5.5), which after averaging over the directions of $\mathbf{p}$ yields

$$
\int \mathrm{d} \Omega_{\mathbf{p}}\left[(K \cdot P)^{2}\right]_{P}=p^{2}\left(\frac{k^{2}}{3-2 \epsilon}-k_{n}^{2}\right) .
$$

A physical interpretation for this structure is given after eq. (8.8).

\section{Next-to-leading order results}

If we choose the renormalization scale as $\bar{\mu}=M$ in eq. (6.9); denote the corresponding renormalized coupling by $\left|h_{\nu}\right|^{2}$; set $N_{\mathrm{c}}=3$; and insert eq. (5.2) together with

$$
\int_{\mathbf{p}} p n_{\mathrm{B}}=\frac{\pi^{2} T^{4}}{30}, \quad \int_{\mathbf{p}} p n_{\mathrm{F}}=\frac{7 \pi^{2} T^{4}}{240}
$$

then the "decay rate" from eq. (2.3) becomes

$$
\begin{aligned}
\Gamma(\mathcal{K})=\frac{\left|h_{\nu}\right|^{2} M^{2}}{8 \pi \sqrt{k^{2}+M^{2}}}\left\{1-\frac{\lambda T^{2}}{M^{2}}-\left|h_{t}\right|^{2}\left[\frac{21}{2(4 \pi)^{2}}+\frac{7 \pi^{2}}{60}\left(\frac{T^{4}}{M^{4}}+\frac{4}{3} \frac{k^{2} T^{4}}{M^{6}}\right)\right]\right. \\
\left.+\left(g_{1}^{2}+3 g_{2}^{2}\right)\left[\frac{29}{8(4 \pi)^{2}}-\frac{\pi^{2}}{80}\left(\frac{T^{4}}{M^{4}}+\frac{4}{3} \frac{k^{2} T^{4}}{M^{6}}\right)\right]+\mathcal{O}\left(g^{4}, \frac{g^{3} T^{2}}{M^{2}}, \frac{g^{2} T^{6}}{M^{6}}\right)\right\} .
\end{aligned}
$$

This constitutes our main result. (The $\mathcal{O}\left(g^{3}\right)$ correction is worked out in section 8 and amounts to $\lambda T^{2} \rightarrow \lambda T\left(T-3 m_{\mathrm{H}} / \pi\right)$, where $m_{\mathrm{H}}$ is the thermal mass parameter given in eq. (8.5).)

Equation (7.2) can be compared with ref. [7]. We completely agree on all $T$-independent terms, as well as on the fact that gauge corrections lead to no term proportional to $T^{2}$. As far as the Higgs contribution is concerned, we find a result larger by a factor 2. As far as the top correction is concerned, we find that all terms of $\mathcal{O}\left(T^{2}\right)$ cancel like in gauge corrections, whereas in ref. [7] only a partial cancellation was observed, so that a term of $\mathcal{O}\left(T^{2}\right)$ was left over. The corrections of $\mathcal{O}\left(T^{4}\right)$ in eq. (7.2) were not considered in ref. [7].

As shown in section 5, we have cross-checked the Higgs contribution by independently computing the full rate for a general $\pi T / M$ and taking the non-relativistic limit only afterwards, cf. eq. (5.16). As far as we can judge, the Higgs correction in ref. [7] was inferred from the effect that a thermal mass would have on a vacuum-like result; but since there are no IR issues at NLO, results emerge from momenta $p \gg g T$, and must be insensitive to thermal masses. In fact, taking a zero-temperature decay rate $\sim M^{2}-m_{0}^{2}$, with $m_{0}^{2}$ denoting a vacuum mass, and denoting by $m_{\mathrm{H}}^{2}=\frac{\lambda T^{2}}{2}$ the thermal mass, then sections 5.2 and 5.3 show that there are two contributions, amounting to $M^{2}-m_{0}^{2} \rightarrow M^{2}-m_{0}^{2}-m_{\mathrm{H}}^{2}-m_{\mathrm{H}}^{2} \frac{\mathrm{d}}{\mathrm{d} m_{0}^{2}} m_{0}^{2}$, 
rather than a single mass shift $\exp \left(m_{\mathrm{H}}^{2} \frac{\mathrm{d}}{\mathrm{d} m_{0}^{2}}\right)\left(M^{2}-m_{0}^{2}\right)$ as was assumed in ref. [7]. One of the contributions comes from a "double-pole" cut and requires a careful analysis.

Let us finally consider the total rather than the differential production rate, obtained according to eq. (2.3) as

$$
\gamma(T) \equiv \frac{\mathrm{d} N}{\mathrm{~d}^{4} \mathcal{X}}=\int \frac{\mathrm{d}^{3} \mathbf{k}}{(2 \pi)^{3}} 2 n_{\mathrm{F}}\left(k^{0}\right) \Gamma(\mathcal{K}) .
$$

A straightforward integration with $\Gamma$ from eq. (7.2) yields

$$
\gamma(T)=\frac{\left|h_{\nu}\right|^{2} M^{3} T}{(2 \pi)^{3}}\left\{\left(1+c_{1}\right) K_{1}\left(\frac{M}{T}\right)+\frac{3 c_{2} T}{M} K_{2}\left(\frac{M}{T}\right)+\mathcal{O}\left[\left(\frac{T}{M}\right)^{\frac{1}{2}} e^{-\frac{2 M}{T}}\right]\right\},
$$

with the coefficients

$$
\begin{aligned}
& c_{1}=-\frac{\lambda T^{2}}{M^{2}}-\left|h_{t}\right|^{2}\left[\frac{21}{2(4 \pi)^{2}}+\frac{7 \pi^{2} T^{4}}{60 M^{4}}\right]+\left(g_{1}^{2}+3 g_{2}^{2}\right)\left[\frac{29}{8(4 \pi)^{2}}-\frac{\pi^{2} T^{4}}{80 M^{4}}\right], \\
& c_{2}=-\left|h_{t}\right|^{2} \frac{7 \pi^{2} T^{4}}{45 M^{4}}-\left(g_{1}^{2}+3 g_{2}^{2}\right) \frac{\pi^{2} T^{4}}{60 M^{4}} .
\end{aligned}
$$

Uncertainties are like in eq. (7.2), except that $c_{2}$ has only thermal corrections. Embedding eq. (7.4) in cosmology, the number density, $n(T) \equiv \frac{\mathrm{d} N}{\mathrm{~d}^{3} \mathrm{x}}$, conventionally normalized to the total entropy density, $s(T)$, evolves as ${ }^{5}$

$$
T \frac{\mathrm{d}}{\mathrm{d} T}\left(\frac{n(T)}{s(T)}\right)=-\frac{\gamma(T)}{3 c_{s}^{2}(T) s(T) H(T)},
$$

where $H(T)$ is the Hubble parameter. This equation is correct as long as the differential abundance remains below its equilibrium value at each $\mathbf{k}$ separately, cf. e.g. ref. [19]; otherwise a "back reaction" from Pauli exclusion needs to be included.

\section{OPE representation}

In section 5, it was mentioned that if the Euclidean correlator $\Pi_{E}$ is considered, then some of the individual terms appear to be IR divergent. As pointed out in ref. [15], the nature of these divergences can be understood by representing the result in an OPE form.

We note first that, carrying out a naive dimensionally regularized NLO computation, the (bare) condensate $\left\langle\phi^{\dagger} \phi\right\rangle_{T}$ reads

$$
\begin{aligned}
\left\langle\phi^{\dagger} \phi\right\rangle_{T}^{\text {naive }}= & 2 \mathcal{J}_{\mathrm{a}}-12 \lambda_{\mathrm{B}} \mathcal{I}_{\mathrm{c}}+2\left|h_{t \mathrm{~B}}\right|^{2} N_{\mathrm{c}}\left(2 \widetilde{\mathcal{I}}_{\mathrm{c}}-\widetilde{\mathcal{I}}_{\mathrm{e}}\right) \\
& +\left(g_{1 \mathrm{~B}}^{2}+3 g_{2 \mathrm{~B}}^{2}\right)\left(\frac{1-D}{2} \mathcal{I}_{\mathrm{c}}+\mathcal{I}_{\mathrm{e}}\right)+\mathcal{O}\left(g^{4}\right) .
\end{aligned}
$$

Here appear the same IR divergent structures as were encountered in section 5 . If we now write $\Pi_{E}=\Pi_{E}^{(0)}+\Pi_{E}^{(T)}$, where $\Pi_{E}^{(0)}$ denotes the vacuum part, then by inserting the

\footnotetext{
${ }^{5}$ The speed of sound squared, $c_{s}^{2}$, is often approximated as $\frac{1}{3}$, which is true in a conformally symmetric situation, but not when mass thresholds are crossed or effects from running couplings are taken into account.
} 
expansions from appendix $\mathrm{C}$ into eqs. (6.1)-(6.6) and renormalizing according to eq. (6.7), the thermal part can be expressed as

$$
\Pi_{E}^{(T)}(K)=-2\left|h_{\nu}\right|^{2}\left[1+\frac{3 \lambda}{8 \pi^{2}}\left(\ln \frac{\bar{\mu}^{2}}{K^{2}}+1+\mathcal{O}(\epsilon)\right)+\mathcal{O}\left(g^{4}\right)\right] \mathcal{Z}_{m} \mu^{2 \epsilon}\left\langle\phi^{\dagger} \phi\right\rangle_{T}^{\text {naive }}+\mathcal{O}\left(\frac{T^{4}}{K^{2}}\right)
$$

where

$$
\mathcal{Z}_{m} \equiv \mathcal{Z}_{\nu}\left[1+\frac{3 \lambda}{8 \pi^{2} \epsilon}+\mathcal{O}\left(g^{4}\right)\right]=1+\frac{1}{(4 \pi)^{2} \epsilon}\left[6 \lambda+\left|h_{t}\right|^{2} N_{\mathrm{c}}-\frac{3}{4}\left(g_{1}^{2}+3 g_{2}^{2}\right)\right]+\mathcal{O}\left(g^{4}\right)
$$

happens to be the renormalization factor related to the Higgs mass parameter, $m_{\mathrm{B}}^{2}=m_{0}^{2} \mathcal{Z}_{m}$. The prefactor in eq. (8.2) is IR-safe (temperature independent) as is typical of a Wilson coefficient; all IR-sensitive terms of $\mathcal{O}\left(T^{2}\right)$ have been eaten up by the condensate.

In order to compute the condensate correctly at $\mathcal{O}\left(g^{2}\right)$ also in the IR, we need to add to eq. (8.1), which in this naive form represents an ultraviolet contribution from "hard" momenta $p \gtrsim \pi T$, the contribution from "soft" momenta, in particular from Matsubara zero modes. This type of computations are best formulated within dimensionally reduced effective field theories. In fact the result can be extracted e.g. from ref. [29], and reads

$$
\begin{aligned}
\left\langle\phi^{\dagger} \phi\right\rangle_{T}^{(n=0)}=-\frac{m_{\mathrm{H}} T}{2 \pi}+\frac{T^{2}}{(4 \pi)^{2}}[ & 6 \lambda+\frac{g_{1}^{2} m_{\mathrm{D} 1}+3 g_{2}^{2} m_{\mathrm{D} 2}}{4 m_{\mathrm{H}}} \\
& \left.+\left(g_{1}^{2}+3 g_{2}^{2}\right)\left(\frac{1}{4 \epsilon}+\ln \frac{\bar{\mu}}{2 m_{\mathrm{H}}}+\frac{1}{4}\right)\right]+\mathcal{O}\left(g^{3}\right),
\end{aligned}
$$

where the Standard Model thermal masses read, for $N_{\mathrm{c}}=3$,

$$
m_{\mathrm{H}}^{2}=m_{0}^{2}+\left(\frac{\lambda}{2}+\frac{\left|h_{t}\right|^{2}}{4}+\frac{g_{1}^{2}+3 g_{2}^{2}}{16}\right) T^{2}, \quad m_{\mathrm{D} 1}^{2}=\frac{11}{6} g_{1}^{2} T^{2}, \quad m_{\mathrm{D} 2}^{2}=\frac{11}{6} g_{2}^{2} T^{2} .
$$

Summing together eqs. (8.1) and (8.4), multiplying by $\mathcal{Z}_{m}$ as required by eq. (8.2), and for convenience also resumming the Debye mass contributions of $\mathcal{O}\left(g^{3}\right)$ into $m_{\mathrm{H}}^{2}$, we get

$$
\begin{aligned}
\mathcal{Z}_{m}\left\langle\phi^{\dagger} \phi\right\rangle_{T}= & \frac{T^{2}}{6}-\frac{T^{2}}{2 \pi} \sqrt{\frac{m_{\mathrm{H}}^{2}}{T^{2}}-\frac{g_{1}^{2} m_{\mathrm{D} 1}+3 g_{2}^{2} m_{\mathrm{D} 2}}{16 \pi T}} \\
& +\frac{T^{2}}{48 \pi^{2}}\left\{-6 \lambda\left[\ln \left(\frac{\bar{\mu} e^{\gamma_{\mathrm{E}}}}{4 \pi T}\right)-3\right]-\left|h_{t}\right|^{2} N_{\mathrm{c}} \ln \left(\frac{\bar{\mu} e^{\gamma_{\mathrm{E}}}}{8 \pi T}\right)\right. \\
& \left.+\frac{3\left(g_{1}^{2}+3 g_{2}^{2}\right)}{4}\left[\ln \left(\frac{\bar{\mu} e^{\gamma_{\mathrm{E}}}}{4 \pi T}\right)-\frac{2}{3}-2 \gamma_{\mathrm{E}}-\frac{2 \zeta^{\prime}(-1)}{\zeta(-1)}+4 \ln \left(\frac{2 \pi T}{m_{\mathrm{H}}}\right)\right]\right\}+\mathcal{O}\left(g^{3}\right) .
\end{aligned}
$$

Apart from eqs. (5.2), (5.3), the fermionic $\int_{\mathbf{p}} \frac{n_{\mathrm{F}}(p)}{p}=\frac{T^{2} \mu^{-2 \epsilon}}{24}\left\{1+2 \epsilon\left[\ln \frac{\bar{\mu}}{8 \pi T}+1+\frac{\zeta^{\prime}(-1)}{\zeta(-1)}\right]\right\}$ was needed here. All ultraviolet divergences have nicely cancelled out in eq. (8.6).

The thermal correction to the "decay rate" of eq. (2.3) now comes from the cut of the Wilson coefficient in eq. (8.2). It is given by

$$
\Gamma^{(T)}(\mathcal{K})=-\frac{3\left|h_{\nu}\right|^{2}}{4 \pi \sqrt{k^{2}+M^{2}}}\left[\lambda+\mathcal{O}\left(g^{4}\right)\right] \mathcal{Z}_{m}\left\langle\phi^{\dagger} \phi\right\rangle_{T}+\mathcal{O}\left(\left|h_{\nu}\right|^{2} g^{2} T^{4}\right) .
$$


Looking back at eq. (8.6), we observe that there is a subleading $\mathcal{O}\left(g^{3}\right)$ contribution to the decay rate (from the term $-\frac{m_{\mathrm{H}} T}{2 \pi} \sim \mathcal{O}(g)$ in $\mathcal{Z}_{m}\left\langle\phi^{\dagger} \phi\right\rangle_{T}$ ) which is sensitive to thermal resummations, however in a computable way.

Let us finally briefly remark on the terms of $\mathcal{O}\left(T^{4}\right)$ in eq. (8.7). As was pointed out in ref. [15], these contain expectation values of various components of the energy-momentum tensor. At finite temperatures, the most important contribution turns out to emerge from its traceless part: denoting $\left\langle\Theta^{\mu \nu}\right\rangle_{T}=\operatorname{diag}(e, p, p, p)$, we may define

$$
\begin{aligned}
\left\langle\hat{\Theta}^{\mu \nu}\right\rangle_{T} & \equiv\left\langle\Theta^{\mu \nu}-\frac{1}{4} \eta^{\mu \nu} \Theta^{\alpha}{ }_{\alpha}\right\rangle_{T}=\operatorname{diag}(e, p, p, p)-\frac{1}{4} \operatorname{diag}(+---)(e-3 p) \\
& =\frac{3}{4}(e+p) \operatorname{diag}\left(1, \frac{1}{3}, \frac{1}{3}, \frac{1}{3}\right) .
\end{aligned}
$$

Since $e+p=T s$, where $s$ is the entropy density, this condensate vanishes at zero temperature. The non-trivial momentum dependence in eq. (6.9) comes from terms of the type $\sum_{i} \mathcal{K}_{\mu} \mathcal{K}_{\nu}\left\langle\hat{\Theta}_{i}^{\mu \nu}\right\rangle_{T} / \mathcal{K}^{6}$, just like in ref. [15], where $\hat{\Theta}_{i}^{\mu \nu}$ denote various gauge-invariant subparts of the full tensor (we have not worked out the decomposition because it is not needed here). Thermal resummations affect $e$ and $p$ first at $\mathcal{O}\left(g^{3} T^{4}\right)[30]$, and thus $\Gamma^{(T)}(\mathcal{K})$ at $\mathcal{O}\left(g^{5} T^{4}\right)$.

\section{Conclusions}

The purpose of this paper has been to compute the thermal production rate of right-handed neutrinos in the non-relativistic regime, meaning at temperatures much below their mass, but still higher than the electroweak scale: $m_{\text {top }} \lesssim \pi T \ll M$. (Equivalently, one can speak of a decay rate, cf. eq. (2.4).) In this regime, the results can be organized in the form of an Operator Product Expansion, with successively higher powers of $\pi T$ representing thermal expectation values of gauge-invariant condensates [15]. We have worked at NLO in the coupling constants (even at NNLO in section 8), and to the third order in an expansion in $(\pi T / M)^{2}$; the main result is shown in eq. (7.2). At order $(\pi T / M)^{0}$ we fully confirm previous NLO results in the literature [7], if employing naive dimensional regularization for handling $\gamma_{5}$; at order $(\pi T / M)^{2}$ we confirm the absence of corrections from gauge bosons, but find different results for corrections from the Higgs scalar and the top quark. Our results of $(\pi T / M)^{4}$ are new, and display the leading non-trivial dependence on the spatial momentum (the terms proportional to $k^{2}$ in eq. (7.2), which contribute at $(\pi T / M)^{5}$ to the total production rate).

Despite small differences, the numerical magnitude of our corrections is similar to what was found in ref. [7]. Even though our NLO thermal Higgs correction is larger by a factor 2, we also find an NNLO term, cf. eq. (8.6), which numerically cancels about half of the Higgs correction. The most substantial difference is that we find a complete cancellation of top corrections at $\mathcal{O}(\pi T / M)^{2}$. Therefore our thermal corrections are negative, cf. eqs. (7.2) and (8.7), not positive as was found for the top correction in ref. [7]. Their precise numerical influence on leptogenesis computations remains to be inspected but probably the effects are no larger than was found in ref. [7] because the largest (top) term is absent at $\mathcal{O}(\pi T / M)^{2}$. 
In scenarios of $\mathrm{TeV}$ scale leptogenesis, the rate that we have computed could be relevant down to temperatures around the electroweak scale. Remarkably, eq. (8.7) shows that then the rate could be extracted non-perturbatively from Euclidean lattice simulations of the type that were developed for studying the electroweak phase transition in the 1990s. In addition eq. (8.7) shows that terms of $\mathcal{O}(\pi T / M)^{2}$ are necessarily proportional to $\lambda$; this can be traced back to a mismatch of the renormalization factors of the neutrino Yukawa coupling (eq. (6.8)) and the Higgs mass parameter (eq. (8.3)) which ultimately leads to the logarithm in eq. (8.2).

As an outlook, we envisage that taking the representations in eqs. (6.1)-(6.6) as starting points, it is a feasible if hard task to extend the NLO analysis to the relativistic regime, $\pi T \sim M$. Indeed a similar step has previously been taken in QCD, by going from OPEtype results in ref. [16] to relativistic results in ref. [28]. We believe that in the current context it would be important to keep the spatial momentum $\mathbf{k}$ different from zero, which makes the analysis more demanding; still, in principle similar techniques should work.

\section{Acknowledgments}

This work was partly supported by the BMBF under project 06BI9002. M.L. thanks Dietrich Bödeker for useful discussions.

Note added. In the revised version (v3) and Erratum of ref. [7], the results have been corrected and now agree with ours.

\section{A Definitions of master sum-integrals}

Denoting by $P, Q$ bosonic and by $K, R, S$ fermionic Matsubara four-momenta, and employing the usual conventions $\mathscr{E}_{P}$ and $\mathscr{W}_{\{R\}}$ for the corresponding measures, the master sum-integrals entering the computation are defined as follows (in the graphical notation, a dashed line indicates a bosonic propagator, a solid line a fermionic one, a filled blob a squared propagator, and a cross that the momentum appears in the numerator as well):

$$
\begin{aligned}
& \stackrel{\ddots}{\vdots} \mathcal{J}_{\mathrm{a}} \equiv \mathcal{F}_{P} \frac{1}{P^{2}} \\
& \bigodot_{\rightarrow} \tilde{\mathcal{J}}_{\mathrm{a}} \equiv \sum_{\{R\}} \frac{1}{R^{2}} \\
& \bigcup_{\mathrm{b}} \equiv \sum_{P} \frac{K^{2}}{P^{2}(P-K)^{2}} \text {, } \\
& \vdots \begin{array}{c}
\cdots \\
\hdashline
\end{array} \\
& \bigcup^{\prime} \times \tilde{\mathcal{I}}_{\mathrm{b}} \equiv \sum_{P\{R\}} \frac{1}{R^{2} P^{2}(P-K)^{2}} \text {, }
\end{aligned}
$$




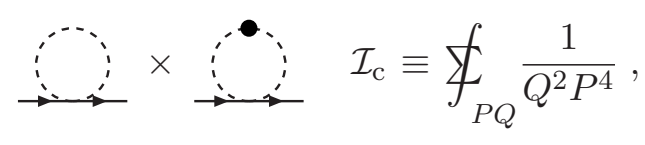

$$
\begin{aligned}
& \bigcirc \times \underset{\vdots}{\vdots} \widetilde{\mathcal{I}}_{\mathrm{c}} \equiv \mathcal{F}_{P\{R\}} \frac{1}{R^{2} P^{4}},
\end{aligned}
$$

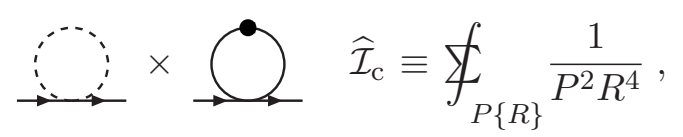

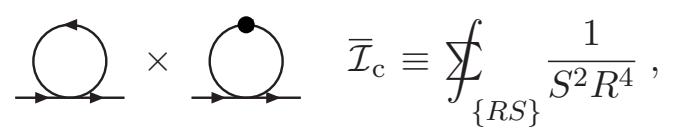

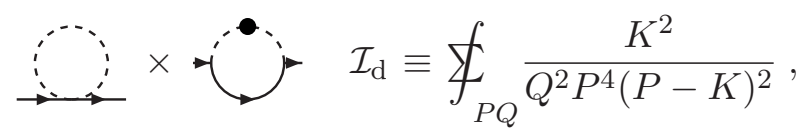

$$
\begin{aligned}
& \bigcirc \times \widetilde{\mathcal{I}}_{\mathrm{d}} \equiv \sum_{P\{R\}} \frac{K^{2}}{R^{2} P^{4}(P-K)^{2}}, \\
& \vdots \quad \cdots \quad \widehat{\mathcal{I}}_{\mathrm{d}} \equiv \oint_{P\{R\}} \frac{K^{2}}{P^{2} R^{4}(R-K)^{2}}, \\
& \bigcirc \times \overline{\mathcal{I}}_{\mathrm{d}} \equiv \mathcal{F}_{\{R S\}} \frac{K^{2}}{S^{2} R^{4}(R-K)^{2}}, \\
& \cdots \quad \mathcal{I}_{\mathrm{e}} \equiv \mathcal{F}_{P Q} \frac{1}{Q^{2} P^{2}(P-Q)^{2}}, \\
& \cdots \widetilde{\mathcal{I}}_{\mathrm{e}} \equiv \sum_{P\{R\}} \frac{1}{R^{2} P^{2}(P-R)^{2}} \\
& \because \mathcal{I}_{\mathrm{f}} \equiv \sum_{P Q} \frac{1}{Q^{2}(Q-P)^{2}(P-K)^{2}}, \\
& \Longrightarrow \widetilde{\mathcal{I}}_{\mathrm{f}} \equiv \oiint_{P\{R\}} \frac{1}{R^{2}(R-P)^{2}(P-K)^{2}}, \\
& \bigcup^{\ddots \cdots} \mathcal{I}_{\mathrm{g}} \equiv \sum_{P Q} \frac{K^{2}}{P^{2}(P-K)^{2} Q^{2}(Q-K)^{2}}, \\
& \text { “ } \mathcal{I}_{\mathrm{h}} \equiv \oiint_{P Q} \frac{K^{2}}{Q^{2} P^{2}(Q-P)^{2}(P-K)^{2}} \text {, } \\
& \text { Y. } \widetilde{\mathcal{I}}_{\mathrm{h}} \equiv \sum_{P\{R\}} \frac{K^{2}}{R^{2} P^{2}(R-P)^{2}(P-K)^{2}} \text {, }
\end{aligned}
$$




$$
\begin{aligned}
& \because \widehat{\mathcal{I}}_{\mathrm{h}} \equiv \sum_{P\{R\}} \frac{K^{2}}{R^{2} P^{2}(R-P)^{2}(R-K)^{2}}, \\
& \because \widehat{\mathcal{I}}_{\mathrm{h}} \equiv \sum_{P\{R\}} \frac{2 K \cdot P}{R^{2} P^{2}(R-P)^{2}(R-K)^{2}}, \\
& \mathcal{I}_{\mathrm{j}} \frac{K^{4}}{Q^{2} P^{2}(Q-P)^{2}(P-K)^{2}(Q-K)^{2}} .
\end{aligned}
$$

\section{B Results for master spectral functions}

The spectral functions corresponding to the structures of appendix A are obtained from

$$
\rho_{\mathcal{I}_{\mathbf{X}}} \equiv \operatorname{Im}\left[\mathcal{I}_{\mathbf{x}}\right]_{k_{n} \rightarrow-i\left[k^{0}+i 0^{+}\right]}
$$

As has been elaborated upon in section 5, they can be derived by carrying out the Matsubara sums, which corresponds to cutting zero, one, or two lines and weighting them with a thermal distribution. In the result, cuts correspond to logarithmic terms, which can only arise from vacuum loops. Given that 2 -cut contributions have no vacuum loop left, spectral functions only arise from 0-cut and 1-cut contributions. After an expansion in small thermal momenta, the vacuum parts always have simple cuts, and thereby the spectral functions corresponding to the master structures of appendix A are elementary, cf. eqs. (B.4)-(B.21) below.

Before presenting the list, we would like to mention that apart from the procedure described in section 5, there exists also a more elaborate possibility for determining the spectral functions. Namely, one can first "blindly" work out the OPE expansion in the Euclidean domain up to the desired order in $1 / K^{2}$. The corresponding results are listed in appendix $\mathrm{C}$. In these results, non-analytic $K$-dependence only appears in a factor $X$, defined in eq. (C.2). Introducing the $\overline{\mathrm{MS}}$ scale parameter, it can be expanded as

$$
X=\frac{\mu^{-2 \epsilon}}{(4 \pi)^{2}}\left(\frac{\bar{\mu}^{2}}{K^{2}}\right)^{\epsilon}\left[1-\frac{\pi^{2} \epsilon^{2}}{12}+\mathcal{O}\left(\epsilon^{3}\right)\right]
$$

and the corresponding spectral function then arises from

$$
\operatorname{Im}[X]_{k_{n} \rightarrow-i\left[k^{0}+i 0^{+}\right]}=\operatorname{sign}\left(k^{0}\right) \frac{\mu^{-2 \epsilon}}{16 \pi}\left[\epsilon+\epsilon^{2} \ln \frac{\bar{\mu}^{2}}{\mathcal{K}^{2}}+\mathcal{O}\left(\epsilon^{3}\right)\right] .
$$

So, we observe that spectral functions can only arise from structures of type $X^{n} / \epsilon^{m}$, and that all Euclidean structures that are either finite or have $1 / \epsilon$-poles with the non-analytic scale dependence given by $T$ rather than by $K$, yield vanishing spectral functions.

Proceeding to the list, we wish to remove clutter by not showing the non-consequential factor $\mu^{-2 \epsilon}$ explicitly, and by similarly omitting the arguments of the functions $n_{\mathrm{B}}, n_{\mathrm{F}}$. In addition, the errors of the 2-loop structures, which are $\mathcal{O}\left(\epsilon, \frac{1}{\mathcal{K}^{4}}\right)$, are not displayed. Thereby 
the spectral functions corresponding to the master structures read

$$
\begin{aligned}
& \rho_{\mathcal{J}_{\mathrm{a}}}=\rho_{\widetilde{\mathcal{J}}_{\mathrm{a}}}=0, \\
& \rho_{\mathcal{J}_{\mathrm{b}}}=-\frac{\mathcal{K}^{2}}{16 \pi}\left[1+\epsilon\left(\ln \frac{\bar{\mu}^{2}}{\mathcal{K}^{2}}+2\right)\right]+\mathcal{O}\left(\epsilon^{2}, e^{-\frac{k^{0}}{T}}\right) \text {, } \\
& \rho_{\mathcal{I}_{\mathrm{b}}}=\int_{\mathrm{p}} \frac{n_{\mathrm{B}}}{16 \pi p}, \\
& \rho_{\widetilde{\mathcal{I}}_{\mathrm{b}}}=-\int_{\mathbf{p}} \frac{n_{\mathrm{F}}}{16 \pi p}, \\
& \rho_{\mathcal{I}_{\mathrm{C}}}=\rho_{\widetilde{\mathcal{I}}_{\mathrm{C}}}=\rho_{\widehat{\mathcal{I}}_{\mathrm{C}}}=\rho_{\overline{\mathcal{I}}_{\mathrm{C}}}=0, \\
& \rho_{\mathcal{I}_{\mathrm{d}}}=-\int_{\mathrm{p}} \frac{n_{\mathrm{B}}}{16 \pi p}, \\
& \rho_{\widetilde{\mathcal{I}}_{\mathrm{d}}}=\int_{\mathbf{p}} \frac{n_{\mathrm{F}}}{16 \pi p}, \\
& \rho_{\widehat{\mathcal{I}}_{\mathrm{d}}}=-\int_{\mathrm{p}} \frac{n_{\mathrm{B}}}{16 \pi p}, \\
& \rho_{\overline{\mathcal{I}}_{\mathrm{d}}}=\int_{\mathbf{p}} \frac{n_{\mathrm{F}}}{16 \pi p}, \\
& \rho_{\mathcal{I}_{\mathrm{e}}}=\rho_{\widetilde{\mathcal{I}}_{\mathrm{e}}}=0 \text {, } \\
& \rho_{\mathcal{I}_{\mathrm{f}}}=\frac{\mathcal{K}^{2}}{8(4 \pi)^{3}}+\int_{\mathbf{p}} \frac{2 n_{\mathrm{B}}-n_{\mathrm{F}}}{16 \pi p}, \\
& \rho_{\widetilde{\mathcal{I}}_{\mathrm{f}}}=\frac{\mathcal{K}^{2}}{8(4 \pi)^{3}}-\int_{\mathbf{p}} \frac{3 n_{\mathrm{F}}}{16 \pi p}, \\
& \rho_{\mathcal{I}_{\mathrm{g}}}=-\frac{\mathcal{K}^{2}}{2(4 \pi)^{3}}\left(\frac{1}{\epsilon}+2 \ln \frac{\bar{\mu}^{2}}{\mathcal{K}^{2}}+4\right)+\int_{\mathbf{p}} \frac{n_{\mathrm{B}}-n_{\mathrm{F}}}{8 \pi p}+\frac{k_{0}^{2}+k^{2} / 3}{2 \pi \mathcal{K}^{4}} \int_{\mathbf{p}} p\left(n_{\mathrm{B}}-n_{\mathrm{F}}\right), \\
& \rho_{\mathcal{I}_{\mathrm{h}}}=-\frac{\mathcal{K}^{2}}{4(4 \pi)^{3}}\left(\frac{1}{\epsilon}+2 \ln \frac{\bar{\mu}^{2}}{\mathcal{K}^{2}}+5\right)-\int_{\mathbf{p}} \frac{2 n_{\mathrm{B}}+n_{\mathrm{F}}}{16 \pi p}-\frac{k_{0}^{2}+k^{2} / 3}{2 \pi \mathcal{K}^{4}} \int_{\mathbf{p}} p\left(\frac{n_{\mathrm{B}}}{3}+\frac{n_{\mathrm{F}}}{2}\right) \text {, } \\
& \rho_{\widetilde{\mathcal{I}}_{\mathrm{h}}}=-\frac{\mathcal{K}^{2}}{4(4 \pi)^{3}}\left(\frac{1}{\epsilon}+2 \ln \frac{\bar{\mu}^{2}}{\mathcal{K}^{2}}+5\right)+\int_{\mathbf{p}} \frac{n_{\mathrm{F}}}{16 \pi p}-\frac{k_{0}^{2}+k^{2} / 3}{2 \pi \mathcal{K}^{4}} \int_{\mathbf{p}} p\left(\frac{n_{\mathrm{F}}}{6}\right), \\
& \rho_{\widehat{\mathcal{I}}_{\mathrm{h}}}=-\frac{\mathcal{K}^{2}}{4(4 \pi)^{3}}\left(\frac{1}{\epsilon}+2 \ln \frac{\bar{\mu}^{2}}{\mathcal{K}^{2}}+5\right)+\int_{\mathbf{p}} \frac{n_{\mathrm{F}}}{16 \pi p}+\frac{k_{0}^{2}+k^{2} / 3}{2 \pi \mathcal{K}^{4}} \int_{\mathbf{p}} p\left(\frac{n_{\mathrm{B}}}{3}+\frac{n_{\mathrm{F}}}{6}\right), \\
& \rho_{\widehat{\mathcal{I}}_{\mathrm{h}}}=-\frac{\mathcal{K}^{2}}{8(4 \pi)^{3}}\left(\frac{1}{\epsilon}+2 \ln \frac{\bar{\mu}^{2}}{\mathcal{K}^{2}}+\frac{9}{2}\right)+\int_{\mathbf{p}} \frac{n_{\mathrm{B}}}{16 \pi p}-\frac{k_{0}^{2}+k^{2} / 3}{2 \pi \mathcal{K}^{4}} \int_{\mathbf{p}} p\left(\frac{n_{\mathrm{F}}}{12}\right), \\
& \rho_{\mathcal{I}_{\mathrm{j}}}=\int_{\mathbf{p}} \frac{n_{\mathrm{F}}-2 n_{\mathrm{B}}}{8 \pi p}+\frac{k_{0}^{2}+k^{2} / 3}{2 \pi \mathcal{K}^{4}} \int_{\mathbf{p}} p\left(\frac{11 n_{\mathrm{F}}}{6}-\frac{7 n_{\mathrm{B}}}{3}\right) \text {. }
\end{aligned}
$$

\section{Euclidean large-momentum expansions}

For completeness, we list here Euclidean large-momentum expansions for the master sumintegrals defined in appendix A. In order to be as concise as possible, we introduce 
the notation

$$
n_{i} \equiv\left\{\begin{array}{rl}
n_{\mathrm{B}}, & \text { bosonic line } \\
-n_{\mathrm{F}}, & \text { fermionic line }
\end{array},\right.
$$

and provide expressions valid simultaneously for all the statistics carried by the lines. The shorthands

$$
X \equiv \frac{K^{-2 \epsilon}}{(4 \pi)^{2-\epsilon}} \frac{\Gamma(1+\epsilon) \Gamma^{2}(1-\epsilon)}{\Gamma(1-2 \epsilon)}, \quad Y \equiv \frac{\Gamma(1+2 \epsilon) \Gamma^{2}(1-2 \epsilon)}{\Gamma(1-3 \epsilon) \Gamma^{2}(1+\epsilon) \Gamma(1-\epsilon)},
$$

as well as

$$
\Theta_{K} \equiv \frac{\frac{k^{2}}{3-2 \epsilon}-k_{n}^{2}}{K^{2}}
$$

are also helpful. Thereby we obtain

$$
\begin{aligned}
& \bigcap^{1}=\int_{\mathbf{p}} \frac{n_{1}(p)}{p} \\
& { }_{2}^{1}=\frac{K^{2} X}{\epsilon(1-2 \epsilon)}+\sum_{i=1}^{2} \int_{\mathbf{p}}\left[\frac{n_{i}(p)}{p}+\frac{4 \Theta_{K} p n_{i}(p)}{K^{2}}\right]+\mathcal{O}\left(\frac{T^{6}}{K^{4}}\right), \\
& { }^{3} \bigcirc \times{ }_{2}^{1} \bigcirc=\frac{X}{\epsilon(1-2 \epsilon)} \int_{\mathbf{p}} \frac{n_{3}(p)}{p}+\frac{1}{K^{2}} \sum_{i=1}^{2} \int_{\mathbf{p}, \mathbf{q}} \frac{n_{3}(p) n_{i}(q)}{p q}+\mathcal{O}\left(\frac{T^{6}}{K^{4}}\right) \text {, } \\
& { }^{1} \times{ }^{2} \bigcirc=\frac{1-2 \epsilon}{2} \int_{\mathbf{p}, \mathbf{q}} \frac{n_{1}(p) n_{2}(q)}{p q^{3}} \text {, } \\
& \bigcirc \times-\frac{X}{\epsilon} \int_{\mathbf{p}} \frac{n_{3}(p)}{p}+\frac{1-2 \epsilon}{2} \int_{\mathbf{p}, \mathbf{q}} \frac{n_{3}(p) n_{1}(q)}{p q^{3}} \\
& +\frac{\epsilon+2(1-\epsilon)(3-2 \epsilon) \Theta_{K}}{(2-\epsilon) K^{2}} \int_{\mathbf{p}, \mathbf{q}} \frac{n_{3}(p) n_{1}(q)}{p q} \\
& +\frac{1}{K^{2}} \int_{\mathbf{p}, \mathbf{q}} \frac{n_{3}(p) n_{2}(q)}{p q}+\mathcal{O}\left(\frac{T^{6}}{K^{4}}\right) \\
& \because \cdots=0=0 \\
& 2=-\frac{K^{2} X^{2} Y}{2 \epsilon(1-2 \epsilon)(1-3 \epsilon)(2-3 \epsilon)} \\
& +\frac{X}{\epsilon(1-2 \epsilon)} \sum_{i=1}^{3} \int_{\mathbf{p}}\left[\frac{n_{i}(p)}{p}+\frac{2 \epsilon(1+\epsilon) \Theta_{K} p n_{i}(p)}{K^{2}}\right] \\
& +\frac{1}{K^{2}} \sum_{i>j} \int_{\mathbf{p}, \mathbf{q}} \frac{n_{i}(p) n_{j}(q)}{p q}+\mathcal{O}\left(\frac{T^{6}}{K^{4}}\right) \\
& =\sqrt[3]{-1}=\frac{K^{2} X^{2}}{\epsilon^{2}(1-2 \epsilon)^{2}} \\
& +\frac{X}{\epsilon(1-2 \epsilon)} \sum_{i=1}^{4} \int_{\mathbf{p}}\left[\frac{n_{i}(p)}{p}+\frac{4 \Theta_{K} p n_{i}(p)}{K^{2}}\right] \\
& +\frac{1}{K^{2}} \int_{\mathbf{p}, \mathbf{q}} \frac{\left(n_{1}+n_{2}\right)(p)\left(n_{3}+n_{4}\right)(q)}{p q}+\mathcal{O}\left(\frac{T^{6}}{K^{4}}\right),
\end{aligned}
$$




$$
\begin{aligned}
& 3^{2}=\frac{K^{2} X^{2} Y}{2 \epsilon^{2}(1-2 \epsilon)(1-3 \epsilon)} \\
& +\frac{X}{\epsilon(1-2 \epsilon)} \int_{\mathbf{p}}\left[\frac{n_{1}(p)}{p}+\frac{2(1+\epsilon)(2+\epsilon) \Theta_{K} p n_{1}(p)}{K^{2}}\right] \\
& -\frac{X}{\epsilon} \sum_{i=3}^{4} \int_{\mathbf{p}}\left[\frac{n_{i}(p)}{p}+\frac{2(1+\epsilon)(2+\epsilon) \Theta_{K} p n_{i}(p)}{3 K^{2}}\right] \\
& +\frac{\epsilon^{2}+4(1-\epsilon)^{2} \Theta_{K}}{\epsilon(2-\epsilon) K^{2}} \int_{\mathbf{p}, \mathbf{q}} \frac{n_{3}(p) n_{4}(q)}{p q} \\
& +\frac{1}{K^{2}} \sum_{i=3}^{4} \int_{\mathbf{p}, \mathbf{q}} \frac{n_{1}(p) n_{i}(q)}{p q}+\mathcal{O}\left(\frac{T^{6}}{K^{4}}\right) \\
& 3_{1}^{2}=\frac{K^{2} X^{2} Y}{2 \epsilon^{2}(1-3 \epsilon)(2-3 \epsilon)} \\
& +\frac{X}{\epsilon(1-2 \epsilon)} \int_{\mathbf{p}}\left[\frac{n_{1}(p)}{p}+\frac{2(1+\epsilon)^{2} \Theta_{K} p n_{1}(p)}{K^{2}}\right] \\
& -\frac{X}{\epsilon} \int_{\mathbf{p}} \frac{2(1+\epsilon) \Theta_{K} p n_{3}(p)}{K^{2}} \\
& +\frac{2 X}{1-2 \epsilon} \int_{\mathbf{p}}\left[\frac{n_{4}(p)}{p}+\frac{(1+\epsilon)\left(1+2 \epsilon^{2}\right) \Theta_{K} p n_{4}(p)}{3 \epsilon K^{2}}\right] \\
& +\frac{\epsilon+2(1-\epsilon)^{2} \Theta_{K}}{\epsilon(2-\epsilon) K^{2}} \int_{\mathbf{p}, \mathbf{q}} \frac{\left(n_{2}+n_{3}\right)(p) n_{4}(q)-n_{2}(p) n_{3}(q)}{p q} \\
& +\frac{2}{K^{2}} \int_{\mathbf{p}, \mathbf{q}} \frac{n_{1}(p) n_{4}(q)}{p q}+\mathcal{O}\left(\frac{T^{6}}{K^{4}}\right), \\
& \left.{ }_{3}^{2}\right)_{4}^{1}=\frac{K^{2} X^{2}(1-Y)}{\epsilon^{3}(1-2 \epsilon)} \\
& -\frac{X}{\epsilon} \sum_{i=1}^{4} \int_{\mathbf{p}}\left[\frac{n_{i}(p)}{p}+\frac{2\left(11+6 \epsilon+\epsilon^{2}\right) \Theta_{K} p n_{i}(p)}{3 K^{2}}\right] \\
& -\frac{2 X(1+\epsilon)}{\epsilon} \int_{\mathbf{p}}\left[\frac{n_{5}(p)}{p}+\frac{(2+\epsilon)(3+\epsilon) \Theta_{K} p n_{5}(p)}{3 K^{2}}\right] \\
& -\frac{\epsilon+2(1-\epsilon)^{2} \Theta_{K}}{\epsilon(2-\epsilon) K^{2}} \int_{\mathbf{p}, \mathbf{q}} \frac{n_{1}(p) n_{2}(q)+n_{3}(p) n_{4}(q)}{p q} \\
& +\frac{\epsilon(1+\epsilon)+6(1-\epsilon)^{2} \Theta_{K}}{\epsilon(2-\epsilon) K^{2}} \sum_{i=1}^{4} \int_{\mathbf{p}, \mathbf{q}} \frac{n_{i}(p) n_{5}(q)}{p q} \\
& +\frac{1}{K^{2}} \int_{\mathbf{p}, \mathbf{q}} \frac{n_{1}(p) n_{3}(q)+n_{2}(p) n_{4}(q)}{p q}+\mathcal{O}\left(\frac{T^{6}}{K^{4}}\right) .
\end{aligned}
$$

\section{On the treatment of Dirac traces}

In analogy with the leading-order example of eq. (4.6), let us compare the NDR expressions in eqs. (6.1)-(6.6) with the recipe explained below eq. (3.6) as well as with the strict 't Hooft - Veltman scheme. After Wick contractions, Lorentz algebra, isospin traces and 
using standard properties of non-chiral Dirac matrices (but doing nothing with $\gamma_{5}$ for the moment), the contributions to the Euclidean correlator $\Pi_{E}(K)$ in Feynman gauge read (omitting the overall factor $\left|h_{\nu \mathrm{B}}\right|^{2}$, and abbreviating Lorentz-indices ${ }^{6} \mu_{1}, \ldots, \mu_{6}$ as in $\gamma_{1} \equiv \gamma_{\mu_{1}}$ or $K_{2} \equiv K_{\mu_{2}}$ etc.)

$$
\begin{aligned}
& \underbrace{\vdots \vdots \vdots}=12 \lambda_{\mathrm{B}} \operatorname{Tr}\left[\gamma_{1} a_{\mathrm{L}} \gamma_{2} a_{\mathrm{R}}\right] \text { f }_{P Q} \frac{K_{1}(K-P)_{2}}{Q^{2} P^{4}(K-P)^{2}} \text {, } \\
& \text { O. }=2\left|h_{t \mathrm{~B}}\right|^{2} N_{\mathrm{c}} \operatorname{Tr}\left[\gamma_{1} a_{\mathrm{L}} \gamma_{2} a_{\mathrm{R}}\right] \operatorname{Tr}\left[\gamma_{3} a_{\mathrm{L}} \gamma_{4} a_{\mathrm{R}}\right] \sum_{P\{R\}} \frac{K_{1}(K-P)_{2} R_{3}(P-R)_{4}}{P^{4}(K-P)^{2} R^{2}(P-R)^{2}} \text {, } \\
& \underbrace{\xi_{n} m^{2}}=\left(g_{1 \mathrm{~B}}^{2}+3 g_{2 \mathrm{~B}}^{2}\right) \operatorname{Tr}\left[\gamma_{1} a_{\mathrm{L}} \gamma_{2} a_{\mathrm{R}}\right] \frac{D}{2} \sum_{P Q} \frac{K_{1}(K-P)_{2}}{Q^{2} P^{4}(K-P)^{2}}, \\
& \underbrace{2}=-\left(g_{1 \mathrm{~B}}^{2}+3 g_{2 \mathrm{~B}}^{2}\right) \operatorname{Tr}\left[\gamma_{1} a_{\mathrm{L}} \gamma_{2} a_{\mathrm{R}}\right] \frac{1}{2} \&_{P Q} \frac{K_{1}(K-P)_{2}(P+Q)^{2}}{P^{4} Q^{2}(P-Q)^{2}(K-P)^{2}}, \\
& \underbrace{}_{1234} \frac{1}{2} \sum_{P Q} \frac{K_{1}(K-P)_{2}(K-Q)_{3}(K-P)_{4}}{P^{2}(P-Q)^{2}(K-P)^{4}(K-Q)^{2}}, \\
& s_{1234} \equiv\left(g_{1 \mathrm{~B}}^{2}+3 g_{2 \mathrm{~B}}^{2}\right)(D-2) \operatorname{Tr}\left[\gamma_{1} a_{\mathrm{L}} \gamma_{6} a_{\mathrm{R}}\right]\left(g_{23} g_{46}-g_{24} g_{36}+g_{34} g_{26}\right) \\
& -g_{1 \mathrm{~B}}^{2} \operatorname{Tr}\left[\gamma_{1} a_{\mathrm{L}} \gamma_{2} \gamma_{6} a_{\mathrm{R}} \gamma_{3} \gamma_{6} a_{\mathrm{R}} \gamma_{4} a_{\mathrm{R}}\right] \\
& -3 g_{2 \mathrm{~B}}^{2} \operatorname{Tr}\left[\gamma_{1} a_{\mathrm{L}} \gamma_{2} \gamma_{6}\left(a_{\mathrm{L}} \gamma_{3} \gamma_{6} a_{\mathrm{L}}-\gamma_{3} \gamma_{6}\right) \gamma_{4} a_{\mathrm{R}}\right] \text {, } \\
& \text { - }=t_{1234} \frac{1}{2} \oint_{P Q} \frac{K_{1}(K-Q)_{2}(P+Q)_{3}(K-P)_{4}}{P^{2} Q^{2}(P-Q)^{2}(K-P)^{2}(K-Q)^{2}}, \\
& t_{1234} \equiv\left(g_{1 \mathrm{~B}}^{2}+3 g_{2 \mathrm{~B}}^{2}\right) \operatorname{Tr}\left[\gamma_{1} a_{\mathrm{L}} \gamma_{6} a_{\mathrm{R}}\right]\left(g_{23} g_{46}-g_{24} g_{36}+g_{34} g_{26}\right) \\
& -3 g_{2 \mathrm{~B}}^{2} \operatorname{Tr}\left[\gamma_{1} a_{\mathrm{L}} \gamma_{2} \gamma_{3} a_{\mathrm{R}} \gamma_{4} a_{\mathrm{R}}\right] \text {. }
\end{aligned}
$$

All diagrams with Higgs self-energy insertions are seen to be proportional to the same structure that already appeared in the LO contribution; if handled as shown below eq. (4.6), they lead to $\operatorname{Tr}\left[\gamma_{1} a_{\mathrm{L}} \gamma_{2} a_{\mathrm{R}}\right] \rightarrow 2 g_{12}$ and immediately reduce to the NDR results. On the other hand, the two diagram classes with the vectors coupling to the lepton line do seem to get additional contributions proportional to different Dirac traces, which have been separated in the second and third lines of eqs. (D.6) and (D.8).

It turns out, however, that upon employing the prescription explained below eq. (3.6) these additional contributions vanish identically, which leaves us with the same results as in NDR for each of the diagrams. As a specific example, consider the second line of eq. (D.8),

$$
\begin{aligned}
8 \operatorname{Tr}\left[\gamma_{1} a_{\mathrm{L}} \gamma_{2} \gamma_{3} a_{\mathrm{R}} \gamma_{4} a_{\mathrm{R}}\right]= & \operatorname{Tr}\left[\gamma_{1} \gamma_{2} \gamma_{3}\left(\gamma_{4}+\gamma_{5} \gamma_{4} \gamma_{5}\right)\right]-\operatorname{Tr}\left[\gamma_{1} \gamma_{5} \gamma_{2} \gamma_{3}\left(\gamma_{4}+\gamma_{5} \gamma_{4} \gamma_{5}\right)\right] \\
& -\operatorname{Tr}\left[\gamma_{1} \gamma_{5} \gamma_{2} \gamma_{3}\left(\gamma_{5} \gamma_{4}+\gamma_{4} \gamma_{5}\right)\right]+\operatorname{Tr}\left[\gamma_{1} \gamma_{2} \gamma_{3}\left(\gamma_{5} \gamma_{4}+\gamma_{4} \gamma_{5}\right)\right] \\
= & \operatorname{Tr}\left[\gamma_{4} \gamma_{1} \gamma_{2} \gamma_{3} \gamma_{5}+\gamma_{1} \gamma_{2} \gamma_{3} \gamma_{4} \gamma_{5}\right]
\end{aligned}
$$

where we have used an anticommuting $\gamma_{5}$ as well as $\gamma_{5}^{2}=1$ in traces with more than one $\gamma_{5}$. Then, for the remaining traces with a single $\gamma_{5}$ we get $4\left(\varepsilon_{4123}+\varepsilon_{1234}\right)$, such that the two

\footnotetext{
${ }^{6}$ Note that we do not use $\mu_{5}$ here, to avoid confusion of $\gamma_{\mu_{5}}$ with $\gamma_{5}$.
} 
terms cancel due to antisymmetry of the $\varepsilon$ tensor. In a completely analogous way, the last two lines of eq. (D.6) are seen not to contribute in this specific scheme.

If the 't Hooft - Veltman scheme is used rather than the recipe below eq. (3.6), then graphs with closed fermion loops do differ from those in NDR. To see this, note that the integral in eq. (D.2) evaluates at zero temperature to

$$
\begin{aligned}
\int_{P R} \frac{K_{1}(K-P)_{2} R_{3}(P-R)_{4}}{P^{4}(K-P)^{2} R^{2}(P-R)^{2}} & =\frac{X^{2} Y K_{1}}{144}\left(\frac{1}{\epsilon^{2}}+\frac{31}{6 \epsilon}+\frac{763}{36}+\mathcal{O}(\epsilon)\right) \\
\times & {\left[4 \epsilon(1-2 \epsilon) \frac{K_{2} K_{3} K_{4}}{K^{2}}+(5+2 \epsilon) K_{2} g_{34}-(1-2 \epsilon)\left(K_{3} g_{24}+K_{4} g_{23}\right)\right] }
\end{aligned}
$$

where the coefficients $X$ and $Y$ are defined in eq. (C.2). With $\operatorname{Tr}\left[\gamma_{3} a_{\mathrm{L}} \gamma_{4} a_{\mathrm{R}}\right] \rightarrow 2 g_{34}$ this yields the NDR result, but with eq. (3.3) we rather have $\operatorname{Tr}\left[\gamma_{3} a_{\mathrm{L}} \gamma_{4} a_{\mathrm{R}}\right]=2 \tilde{g}_{34}$, where $\tilde{g}_{\mu \nu} \equiv 1$, if $\mu=\nu \leq 3$, and $\tilde{g}_{\mu \nu} \equiv 0$ otherwise. Contracting with the $g_{34}$ in eq. (D.10) produces 4 rather than $4-2 \epsilon$, which turns into a difference of $\mathcal{O}(1)$ in the spectral function because of the prefactor $1 / \epsilon^{2}$ (concretely, $\frac{7}{2}$ in eq. (6.9) turns into $\frac{73}{18}$ ). That said, this difference can presumably be "hidden" if the neutrino Yukawa coupling is expressed in terms of a physical quantity (such as a pole mass) through a computation carried out in the same scheme.

In thermal corrections to eq. (D.10), there is only a prefactor $1 / \epsilon$ in the Euclidean domain (cf. appendix $\mathrm{C}$ ), which implies that the corresponding spectral function is finite (cf. appendix B). Therefore we expect that the ambiguity of $\mathcal{O}(\epsilon)$ in a prefactor does not affect NLO thermal corrections to spectral functions. Nevertheless, it might be interesting to work out the full tensor integrals of eqs. (D.1)-(D.7) in the OPE regime, in analogy with the expansions in appendix $\mathrm{C}$, thereby producing results for any desirable scheme. Unfortunately this involves a substantial amount of work and goes beyond the scope of the present study.

\section{References}

[1] F.D. Steffen, Dark matter candidates - Axions, neutralinos, gravitinos and axinos, Eur. Phys. J. C 59 (2009) 557 [arXiv:0811.3347] [inSPIRE].

[2] A. Boyarsky, O. Ruchayskiy and M. Shaposhnikov, The role of sterile neutrinos in cosmology and astrophysics, Ann. Rev. Nucl. Part. Sci. 59 (2009) 191 [arXiv:0901.0011] [INSPIRE].

[3] M. Fukugita and T. Yanagida, Baryogenesis without Grand Unification, Phys. Lett. B 174 (1986) 45 [InSPIRE].

[4] W. Buchmüller, R.D. Peccei and T. Yanagida, Leptogenesis as the origin of matter, Ann. Rev. Nucl. Part. Sci. 55 (2005) 311 [hep-ph/0502169] [INSPIRE].

[5] S. Davidson, E. Nardi and Y. Nir, Leptogenesis, Phys. Rept. 466 (2008) 105 [arXiv: 0802.2962] [INSPIRE].

[6] A. Anisimov, D. Besak and D. Bödeker, Thermal production of relativistic Majorana neutrinos: strong enhancement by multiple soft scattering, JCAP 03 (2011) 042 [arXiv: 1012.3784] [INSPIRE]. 
[7] A. Salvio, P. Lodone and A. Strumia, Towards leptogenesis at NLO: the right-handed neutrino interaction rate, JHEP 08 (2011) 116 [arXiv:1106.2814] [INSPIRE].

[8] M. Garny, A. Hohenegger and A. Kartavtsev, Medium corrections to the CP-violating parameter in leptogenesis, Phys. Rev. D 81 (2010) 085028 [arXiv:1002.0331] [INSPIRE].

[9] M. Beneke, B. Garbrecht, C. Fidler, M. Herranen and P. Schwaller, Flavoured leptogenesis in the CTP formalism, Nucl. Phys. B 843 (2011) 177 [arXiv:1007.4783] [InSPIRE].

[10] C.S. Fong, M. Gonzalez-Garcia and J. Racker, CP violation from scatterings with gauge bosons in leptogenesis, Phys. Lett. B 697 (2011) 463 [arXiv:1010.2209] [INSPIRE].

[11] J.-S. Gagnon and M. Shaposhnikov, Baryon asymmetry of the universe without Boltzmann or Kadanoff-Baym equations, Phys. Rev. D 83 (2011) 065021 [arXiv:1012.1126] [INSPIRE].

[12] A. Anisimov, W. Buchmüller, M. Drewes and S. Mendizabal, Quantum leptogenesis I, Annals Phys. 326 (2011) 1998 [arXiv: 1012.5821] [INSPIRE].

[13] C. Kiessig and M. Plümacher, Hard-thermal-loop corrections in leptogenesis I: CP-asymmetries, arXiv:1111.1231 [INSPIRE].

[14] K.G. Wilson and W. Zimmermann, Operator product expansions and composite field operators in the general framework of quantum field theory,

Commun. Math. Phys. 24 (1972) 87 [INSPIRE].

[15] S. Caron-Huot, Asymptotics of thermal spectral functions, Phys. Rev. D 79 (2009) 125009 [arXiv:0903.3958] [INSPIRE].

[16] M. Laine, M. Vepsäläinen and A. Vuorinen, Ultraviolet asymptotics of scalar and pseudoscalar correlators in hot Yang-Mills theory, JHEP 10 (2010) 010 [arXiv:1008.3263] [INSPIRE].

[17] Y. Schröder, M. Vepsäläinen, A. Vuorinen and Y. Zhu, The ultraviolet limit and sum rule for the shear correlator in hot Yang-Mills theory, JHEP 12 (2011) 035 [arXiv:1109.6548] [INSPIRE].

[18] T. Asaka, M. Laine and M. Shaposhnikov, On the hadronic contribution to sterile neutrino production, JHEP 06 (2006) 053 [hep-ph/0605209] [INSPIRE].

[19] M. Laine and M. Shaposhnikov, Sterile neutrino dark matter as a consequence of $\nu M S M$-induced lepton asymmetry, JCAP 06 (2008) 031 [arXiv:0804.4543] [INSPIRE].

[20] M. Le Bellac, Thermal field theory, Cambridge University Press, Cambridge U.K. (2000).

[21] G. 't Hooft and M.J.G. Veltman, Regularization and renormalization of gauge fields, Nucl. Phys. B 44 (1972) 189 [InSPIRE].

[22] P. Breitenlohner and D. Maison, Dimensional Renormalization and the Action Principle, Commun. Math. Phys. 52 (1977) 11 [inSPIRE].

[23] J.G. Körner, N. Nasrallah and K. Schilcher, Evaluation of the flavor changing vertex $b \rightarrow s H$ using the Breitenlohner-Maison-'t Hooft-Veltman $\gamma_{5}$ scheme, Phys. Rev. D 41 (1990) 888 [INSPIRE].

[24] A.J. Buras and P.H. Weisz, QCD nonleading corrections to weak decays in dimensional regularization and 't Hooft-Veltman schemes, Nucl. Phys. B 333 (1990) 66 [INSPIRE].

[25] S.A. Larin, The renormalization of the axial anomaly in dimensional regularization, Phys. Lett. B 303 (1993) 113 [hep-ph/9302240] [INSPIRE]. 
[26] S.A. Larin and J.A.M. Vermaseren, The $\alpha_{s}^{3}$ corrections to the Bjorken sum rule for polarized electroproduction and to the Gross-Llewellyn Smith sum rule, Phys. Lett. B 259 (1991) 345 [INSPIRE].

[27] P.B. Arnold and O. Espinosa, The effective potential and first order phase transitions: beyond leading-order, Phys. Rev. D 47 (1993) 3546 [Erratum ibid. D 50 (1994) 6662] [hep-ph/9212235] [INSPIRE].

[28] M. Laine, A. Vuorinen and Y. Zhu, Next-to-leading order thermal spectral functions in the perturbative domain, JHEP 09 (2011) 084 [arXiv:1108.1259] [INSPIRE].

[29] K. Farakos, K. Kajantie, K. Rummukainen and M.E. Shaposhnikov, 3D physics and the electroweak phase transition: perturbation theory, Nucl. Phys. B 425 (1994) 67 [hep-ph/9404201] [INSPIRE].

[30] J.I. Kapusta, Quantum chromodynamics at high temperature, Nucl. Phys. B 148 (1979) 461 [INSPIRE]. 